\title{
Nature and Interaction of Signals From the Receptive Field Center and Surround in Macaque V1 Neurons
}

\author{
JAMES R. CAVANAUGH, ${ }^{2}$ WYETH BAIR,,${ }^{1,2}$ AND J. ANTHONY MOVSHON ${ }^{1,2}$ \\ ${ }^{1}$ Howard Hughes Medical Institute and ${ }^{2}$ Center for Neural Science, New York University, New York City, New York 10003
}

Received 17 August 2001; accepted in final form 24 July 2002

\begin{abstract}
Cavanaugh, James R., Wyeth Bair, and J. Anthony Movshon. Nature and interaction of signals from the receptive field center and surround in macaque V1 neurons. J Neurophysiol 88: 2530-2546, $2002 ; 10.1152 /$ jn.00692.2001. Information is integrated across the visual field to transform local features into a global percept. We now know that V1 neurons provide more spatial integration than originally thought due to the existence of their nonclassical inhibitory surrounds. To understand spatial integration in the visual cortex, we have studied the nature and extent of center and surround influences on neuronal response. We used drifting sinusoidal gratings in circular and annular apertures to estimate the sizes of the receptive field's excitatory center and suppressive surround. We used combinations of stimuli inside and outside the receptive field to explore the nature of the surround influence on the receptive field center as a function of the relative and absolute contrast of stimuli in the two regions. We conclude that the interaction is best explained as a divisive modulation of response gain by signals from the surround. We then develop a receptive field model based on the ratio of signals from Gaussian-shaped center and surround mechanisms. We show that this model can account well for the variations in receptive field size with contrast that we and others have observed and for variations in size with the state of contrast adaptation. The model achieves this success by simple variations in the relative gain of the two component mechanisms of the receptive field. This model thus offers a parsimonious explanation of a variety of phenomena involving changes in apparent receptive field size and accounts for these phenomena purely in terms of two receptive field mechanisms that do not themselves change in size. We used the extent of the center mechanism in our model as an indicator of the spatial extent of the central excitatory portion of the receptive field. We compared the extent of the center to measurements of horizontal connections within V1 and determined that horizontal intracortical connections are well matched in extent to the receptive field center mechanism. Input to the suppressive surround may come in part from feedback signals from higher areas.
\end{abstract}

I N T R O D U C T I O N

A neuron in visual cortex receives input from a particular region of the visual field. Within this region is a primary excitatory area traditionally studied in the literature- - the classical receptive field or CRF. Information must be incorporated from distinct and distant regions of the visual field to create a global visual percept from local features. Although this integration has traditionally been attributed to higher visual areas with larger CRFs, it is now known that neurons in primary visual cortex receive signals, typically suppressive, from a

Address for reprint requests: J. A. Movshon, Center for Neural Science, New York University, 4 Washington Place, Rm 809, New York 10003-6621 (E-mail: movshon@nyu.edu). region extending beyond the classical receptive field (Allman et al. 1985; Blakemore and Tobin 1972; DeAngelis et al. 1994; Dreher 1972; Hubel and Wiesel 1968; Levitt and Lund 1997; Nelson and Frost 1985; Sillito et al. 1995). Thus early visual cortical areas such as V1 integrate information from rather large areas of the visual field. To study the limits of early spatial integration, one must determine the nature and extent of the classical central excitatory influence and the more extensive suppressive influence.

Different laboratories have used different methods to measure the CRF. Some used the minimum response field or MRF (Barlow et al. 1967), which estimates the extent of the excitatory influence by marking off the portions of the visual field in which a small edge or bar of light elicits a response from the neuron. Others used expanding patches of drifting grating to estimate the region of summation (DeAngelis et al. 1994; Sceniak et al. 1999). Because the central excitatory region of the receptive field becomes less sensitive farther from the center (Movshon et al. 1978a,b), small isolated stimuli near the insensitive receptive field fringes may only afford subthreshold stimulation that would be undetected using the MRF method but would cause changes in suprathreshold responses in the summation technique. Thus these two methods for measuring receptive field extent typically yield quite different resultsMRF measurements of receptive field size are usually smaller than summation measurements. Moreover, Gilbert et al. (1996) and Kapadia et al. (1999) demonstrated with bar stimuli that summation depends on stimulus contrast, and Sceniak et al. (1999) showed analogous effects that depend on the contrast of grating targets.

Expanding a patch of grating beyond the region of summation often reveals suppression as the stimulus engages the otherwise silent inhibitory surround. Responses to larger stimuli therefore reflect the interaction between excitatory and inhibitory mechanisms with different spatial structure. The challenge we address in this paper is to reconstruct the underlying spatial structure of the excitatory center and inhibitory surround mechanisms and to determine the manner of their interaction. We first show that signals from the surround modulate responses of the center through a divisive gain control. We then construct and test a model based on the ratio of two Gaussian sensitivity distributions that accounts well for variations in receptive field size and suppression evident under

\footnotetext{
The costs of publication of this article were defrayed in part by the payment of page charges. The article must therefore be hereby marked "advertisement" in accordance with 18 U.S.C. Section 1734 solely to indicate this fact.
} 
different measurement conditions. These variations are well captured by a model in which the spatial extents of the center and surround are stable features of the receptive field; their sensitivities depend differently on stimulus contrast and recent stimulation history. Such a model, while not intended to suggest any particular biophysical implementation, reveals simply that changes in the size of a receptive field can be generated by changing only the sensitivities of center and surround mechanisms that are themselves stable in spatial extent. The extent of these regions is substantially larger than previously thought and leads us to propose a novel interpretation of the role of different neuronal circuits in generating visual cortical receptive fields.

\section{METHODS}

\section{Subjects and surgical preparation}

We collected data from simple and complex cells in primary visual cortex of 14 adult Cynomolgus monkeys (Macaca fascicularis) and 5 adult pig-tailed macaques (M. nemestrina). Before surgery, each animal was premedicated with $1.5 \mathrm{mg} / \mathrm{kg}$ diazepam or $0.05 \mathrm{mg} / \mathrm{kg}$ acepromazine maleate and $0.05 \mathrm{mg} / \mathrm{kg}$ atropine sulfate. Each monkey was initially anesthetized with $10.0 \mathrm{mg} / \mathrm{kg}$ ketamine $\mathrm{HCl}$. Anesthesia during surgery was maintained by $1.5-3.5 \%$ halothane or isoflurane in a $98 \% \mathrm{O}_{2}-2 \% \mathrm{CO}_{2}$ mixture. Surgery consisted of the placement of cannulae in the saphenous veins of both legs as well as installation of a tracheal cannula. The monkey's head was then placed in a stereotaxic frame, and a small craniotomy was made over parafoveal opercular V1 in one of two locations. The more medial of the craniotomies was made just posterior to the lunate sulcus and about $10 \mathrm{~mm}$ lateral to the midline. This type of vertical penetration yielded two, and sometimes three, passes through V1: opercular V1 near the fovea, upper-bank calcarine V1 in the lower visual field, and lower-bank calcarine V1 in the upper visual field. The second type of craniotomy was positioned more laterally and yielded a tangential penetration that remained in opercular parafoveal V1. An agar-filled plastic chamber was placed over each craniotomy to prevent cortical desiccation and to reduce pulsations of the cortical surface. The agar in the chamber was topped with petroleum jelly and/or Parafilm to prevent dehydration and shrinkage of the agar.

Anesthesia was maintained for the duration of each experiment with sufentanyl citrate $(4-12 \mu \mathrm{g} / \mathrm{kg} / \mathrm{h})$ in lactated Ringer solution with dextrose $(2.5 \%)$ administered through one of the leg cannulae. The animal was paralyzed with vecuronium bromide (Norcuron, $0.1 \mathrm{mg} / \mathrm{kg}$ ), also in lactated Ringer solution, providing a total hourly fluid infusion of $3.25-15.0 \mathrm{ml} / \mathrm{kg} / \mathrm{h}$, depending on the animal's weight. The state of anesthesia was continually monitored by electrocardio- and electroencephalography (ECG and EEG), which was recorded from two sites separated along the cranial surface. The level of anesthesia was adjusted when necessary by changing the infusion rate of the anesthetic. The animal was artificially respirated with moist room air at 22-30 strokes per minute, with stroke volume adjusted to keep end-tidal $\mathrm{CO}_{2}$ between 30 and $36 \mathrm{mmHg}$. Body temperature was maintained at or near $37.5^{\circ} \mathrm{C}$ by a thermostatically controlled heating pad attached to a rectal temperature probe. The pupils were dilated with topical atropine sulfate, and the corneas were protected by clear contact lenses $(+2.0$ diopters). Corrective lenses were used as needed to make the retinae conjugate with the display screen, as determined initially by direct ophthalmoscopy and later by maximizing neuronal responses to sinusoidal gratings at high spatial frequencies. The locations of both foveae were plotted on a tangent screen that was also used for mapping receptive fields.

\section{Visual stimulation}

Visual stimuli were generated by a Truevision ATVista board or a Cambridge Research Systems VSG 2/2 graphics board and displayed on a Nanao T560i monitor (mean luminance: $33 \mathrm{~cd} / \mathrm{m}^{2}$, frame rate: $106 \mathrm{~Hz}$, subtense: $8-25^{\circ}$ visual angle depending on viewing distance). Nonlinearities in phosphor output were corrected by lookup tables.

Sinusoidal gratings were presented alone or in conjunction with another grating (or another pair of gratings) on a gray background with the same mean luminance as the stimulus. The screen contained a mean gray field during inter-stimulus intervals. Stimuli within the classical receptive field (CRF) were contained in a circular window, while stimuli outside the $\mathrm{CRF}$ were in an annular window surrounding the CRF or in circular windows outside the CRF. Stimulus windows had rectangular contrast profiles. Simultaneous gratings were sometimes presented by temporally interleaving the two component stimuli in alternate frames, resulting in each grating appearing at half the maximum available contrast (50\%). Except in these cases, and when contrast was an experimental variable, gratings were presented at $100 \%$ contrast. Simultaneous gratings had the same spatial and temporal frequency but could have their contrasts independently varied. When we presented compound center/surround gratings, stimulus onset of both component gratings was always synchronous.

\section{Unit recording and analysis}

We recorded neural activity using tungsten-in-glass electrodes (Merrill and Ainsworth 1972), the initial signals of which were amplified, band-pass filtered, and fed into a time-amplitude window discriminator (Bak Instruments). Action potential pulses from the window discriminator and synchronization pulses from the graphics board were both collected by a computer interface (Cambridge Electronic Design 1401 Plus) and stored with a resolution of $0.25 \mathrm{~ms}$. We measured cell response as either the mean response firing rate minus the mean spontaneous firing rate (for complex cells) or the magnitude of the first harmonic response (for simple cells) at the temporal frequency of drift. Cell class (simple or complex) was determined for each neuron based on which measure of response (mean firing rate or first harmonic response) provided the greatest value during determination of spatial frequency tuning (Skottun et al. 1991).

\section{Experimental design}

Each experiment consisted of a number of different stimuli pseudorandomly ordered in blocks, the number of blocks determining the number of times each stimulus was repeated. Within each block, each stimulus was presented for 1.5-6 s, and experiments contained from two to five blocks. The inter-stimulus interval was typically about $2 \mathrm{~s}$ but could be as long as $5 \mathrm{~s}$ depending on the time required for the software to generate stimuli of higher complexity.

Receptive fields were initially mapped by hand on a tangent screen, the position and the dimensions of the receptive field being qualitatively determined by listening to the discharge on the audio monitor. We then occluded the nonpreferred eye and used a front-surface mirror to center the receptive field on the monitor. After a brief qualitative determination of the preferred orientation, spatial frequency, and temporal frequency, quantitative assessment of tuning characteristics commenced under computer control.

For each neuron, we first determined the preferred orientation and direction. This was done quantitatively by measuring the response to sinusoidal gratings drifting in different directions, centered on the receptive field as determined by initial mapping. We then determined the preferred spatial frequency by presenting drifting gratings at the neuron's preferred orientation while varying spatial period. Finally, we determined the preferred temporal frequency in a similar manner, 
using stimuli with the neuron's preferred orientation and spatial frequency.

\section{Histology}

At the end of experiments, animals were perfused with $4 \%$ paraformaldehyde in saline. The brains were blocked either parasagittally or coronally, and blocks of tissue were cryoprotected by sinking them in a series of sucrose solutions of increasing concentration (10-30\%). Blocks were cut into $40-\mu \mathrm{m}$ sections that were mounted on slides and stained for Nissl substance with cresyl violet.

We reconstructed electrode tracks from cortical slices of each animal by locating lesions made by passing small amounts of current ( $2 \mu \mathrm{A}, 2-5 \mathrm{~s})$ through the electrode tip and visualizing tissue damage from the passage of the electrode. We confirmed track locations through cortex by comparing them with depths of gray matter/white matter transitions that were noted during each penetration. We determined the laminar location of each cell by overlaying a mosaic image of histological sections with a scaled plot of cell depths along the electrode penetration. Cells were assigned to laminae based on visual inspection of landmarks in the stained sections.

\section{Model fitting}

When comparing neuronal responses to model predictions, we used the STEPIT algorithm (Chandler 1965) to minimize the combined $\chi^{2}$ errors between recorded response magnitudes and model predictions. When a family of curves was recorded, all curves in a family were simultaneously fit, and the minimization algorithm chose the best values for parameters that were not permitted to vary among curves.

We assessed the goodness of each fit by calculating the $\chi^{2}$ error between the data and the model predictions

$$
\chi^{2}=\sum_{i} \frac{\left(e_{i}-o_{i}\right)^{2}}{\sigma_{i}^{2}}
$$

where $e_{i}$ was the model's expectation of response for the $i$ th stimulus, $o_{i}$ was the observed response, and $\sigma_{i}^{2}$ was the expected variance of the response.

To avoid unreasonably large errors from randomly small measures of variance, we exploited the observation that the relationship between the variance and mean of cortical neural responses is linear (e.g., Schiller et al. 1976; Tolhurst et al. 1981, 1983; Vogels et al. 1989). We used neuronal spike counts from the pooled responses of each neuron to calculate the constant of proportionality of response variance to response rate- the variance to mean ratio $(\rho)$-for each cell. We then used this ratio to compute the expected variance for each response, thus discounting random fluctuations in variance that could cause inflated error calculations. For simple cells, means and variances were calculated accounting for response phase. $\chi^{2}$ error was taken as

$$
\chi^{2}=\sum \frac{(e-o)^{2}}{k+o \cdot \frac{\rho}{t}}
$$

in which $o$ is the observed response rate, $e$ is the expected (fit) response rate, $\rho$ is the variance to mean ratio, $t$ is response duration (required to convert the variance of spike counts to the variance of the response rate), and $k$ is a small factor, calculated for each cell, to prevent responses of zero from producing infinite errors $[k=$ $0.01(\rho \max (o))]$.

This raw $\chi^{2}$ is not appropriate for comparing models with different numbers of free parameters because of the different numbers of degrees of freedom. To compare fits for models with different num- bers of degrees of freedom, we used the normalized $\chi^{2}$ value, $\chi_{N}^{2}$ (Hoel et al. 1971):

$$
\chi_{N}^{2}=\frac{\chi^{2}}{d f}
$$

where $d f$ was the number of degrees of freedom in the model.

\section{R E S U L T S}

We recorded the responses of 352 neurons in $\mathrm{V} 1$. We only included neurons in our analysis that fired at least five spikes/s (334/352 units), and we excluded neurons for which we could not determine the CRF boundaries (see following text, 29/334 units). Fifty-seven percent of receptive fields in our sample were centered within $5^{\circ}$ of the fovea, with an additional $9 \%$ between 5 and $10^{\circ}$. Eccentricities between 10 and $25^{\circ}$ accounted for $19 \%$ of our data, and the remaining $15 \%$ of receptive fields had eccentricities between 25 and $40^{\circ}$. Simple and complex cells did not respond differently in our experiments, and have been pooled for all analyses.

\section{Spatial distribution of excitatory and suppressive influences}

We obtained estimates of CRF extent, surround extent, and surround suppression from stimulus expansion tuning curves. Figure $1 A(0)$ shows an example of a stimulus expansion tuning curve for a simple cell. The response of the neuron is plotted as a function of grating patch diameter. Patches of drifting grating were centered on the CRF and presented at the neuron's preferred orientation, spatial and temporal frequencies. The diameter of a patch of grating was systematically varied over a range of eight or nine logarithmically spaced values. Stimulus diameters ranged from 0.15 to $15.7^{\circ}$ of visual angle. Diameter tuning curves followed a typical pattern, of which Fig. $1 A(\mathbf{O})$ is representative. For very small stimuli, responses were low. Responses increased with stimulus diameter and were suppressed for the largest stimuli. For our analysis, we took the grating summation field (GSF) as the diameter of the smallest stimulus that elicited at least $95 \%$ of the neuron's maximum response ( $1.3^{\circ}$ in this example).

Stimulation by the large grating patches usually caused a measurable reduction in response. Suppression increased as the stimulus continued to extend into the receptive field periphery until further expansion into the surround no longer produced additional suppression. We took the inhibitory surround extent as the diameter of the smallest stimulus for which the neuron's response was reduced to within $5 \%$ of its asymptotic value for the largest gratings. For 29 of 334 units, there was neither suppression nor response saturation for our largest stimuli, and we were therefore unable to estimate the extent of the CRF. An example of such a data set is drawn in Fig. 1C. These cells were not included in further analyses. For some other neurons (123/305 units), the suppression saturation point was not reached (Fig. 1D). For these cells, we considered the extent of the surround to be the diameter of the largest stimulus presented. We occasionally observed nonmonotonic suppressive effects (De Valois et al. 1985), but the magnitudes of these effects and their frequency of occurrence were not substantial enough to affect our primary findings. 
A

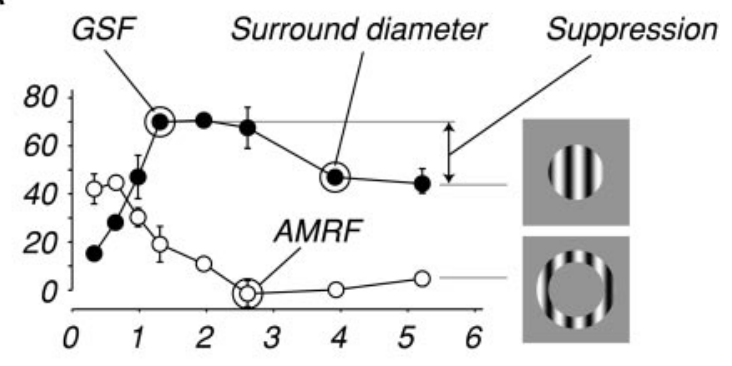

B

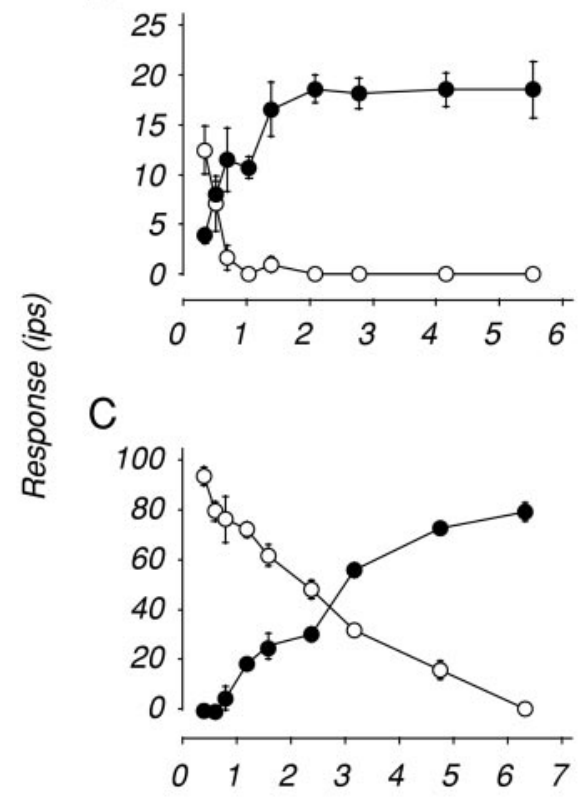

D

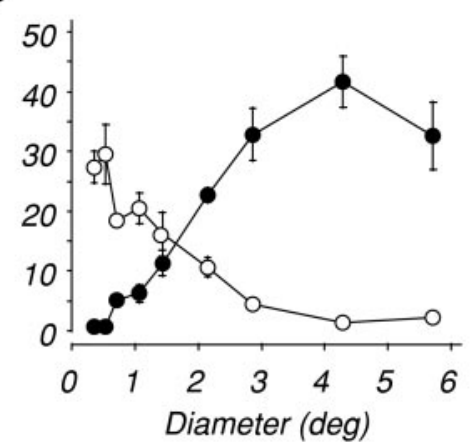

FIG. 1. Example diameter tuning curves for circular and annular patches of grating. Responses of 4 neurons are shown to patches of circular grating $(\bullet)$ and annular patches of grating (O). A: typical diameter tuning curves. The smallest diameter at which responses to circular patches stopped increasing was our initial measure of receptive field extent, the grating summation field (GSF). The smallest inner diameter at which the neuron ceased responding to the annular stimulus was the annular minimum response field (AMRF). We extracted the diameter of the surround from our data as the smallest circular patch diameter at which the suppressive influence asymptoted. Suppression was the relative reduction in response from the neuron's maximum response to this asymptotic response. For some neurons $(B-D)$, we were unable to extract all parameters because there was no suppression $(B)$, no response saturation $(C)$, or no suppression asymptote $(D)$.
Surround suppression strength for each neuron was calculated from the diameter tuning curve as the reduction from the maximum response to the asymptotic response for large stimuli. We computed a suppression index, SI, which expressed suppression as a fraction of the optimal response

$$
\mathrm{SI}=\frac{R_{\mathrm{opt}}-R_{\mathrm{supp}}}{R_{\mathrm{opt}}}
$$

where $R_{\text {opt }}$ is the maximum response, and $R_{\text {supp }}$ is the suppressed response (Fig. 1A).

In most cases, the patch diameter tuning experiment included a second set of stimuli, consisting of drifting gratings in an annular window centered on the receptive field. The outer diameter of the annulus was fixed at the largest value possible on our display, whereas the inner diameter assumed the same values as the circular patch diameters. Figure 1, $\bigcirc$, shows responses to the annular stimuli as a function of increasing inner diameter. Responses to annuli with the smallest inner diameters (leftmost $\bigcirc$ ) approximated responses to the largest circular patches of grating (rightmost O), as expected. Progressing from left to right in the plot, the inner edge of the annulus withdrew from the center of the CRF, and the response of the neuron decreased, eventually reaching the spontaneous rate when the CRF was no longer stimulated. We estimated the extent of the CRF as the point at which the response to the annular stimulus reached a value of at most $5 \%$ of the neuron's maximum response to a circular patch of grating. We called this estimate the annular minimum response field or AMRF.

We compared the empirically derived extents of the center and surround influences for the 260 neurons (of 305) for which suppression was greater than $10 \%$. Figure 2 shows the distribution of GSF diameters and surround diameters for four different ranges of eccentricities (cf. Levitt and Lund 2002). The average GSF diameter for small eccentricities (eccentricity $\mu=2.4^{\circ}, \sigma=1.2^{\circ}$ ) was $0.8^{\circ}$ and increased to $2.1^{\circ}$ for the largest eccentricities (eccentricity $\mu=29.6^{\circ}, \sigma=2.8^{\circ}$ ). These means were significantly different ( $t$-test, $P \ll 0.001)$. The average surround diameter for low eccentricities was $2.5^{\circ}$ and increased to $6.9^{\circ}$ for the largest eccentricities. These means were also significantly different ( $t$-test, $P \ll 0.001)$. The distributions cumulated along the diagonal of each panel in Fig. 2 show the ratios of surround to GSF extent. These distributions do not differ from one another, and over all eccentricities the geometric mean ratio was $3.2 \pm 2.0$ (mean \pm SD) (cf. Li and Li 1994; Maffei and Fiorentini 1976).

Examining distributions of GSF diameter and surround diameter by cortical lamina showed slightly larger receptive field diameters in layer 6 , while surrounds appeared smaller for layer 2/3 neurons. Despite visible trends, homogeneity for these distributions could not be rejected on the basis of a $\chi^{2}$ test. Sceniak et al. (2001) observed significantly larger receptive field sizes in layer 6 and smaller receptive fields in layer $3 B$. They also showed that layer $2 / 3$ a surrounds were smaller, although not significantly. They did observe, however, that layer 2/3a surrounds were significantly smaller than layer 6 surrounds. As our penetrations often went through the operculum into the calcarine sulcus, we were unable to use a canonical depth grid for unbiased layer placement and instead had to rely on visual inspection of electrode tracks (see METHODS). 


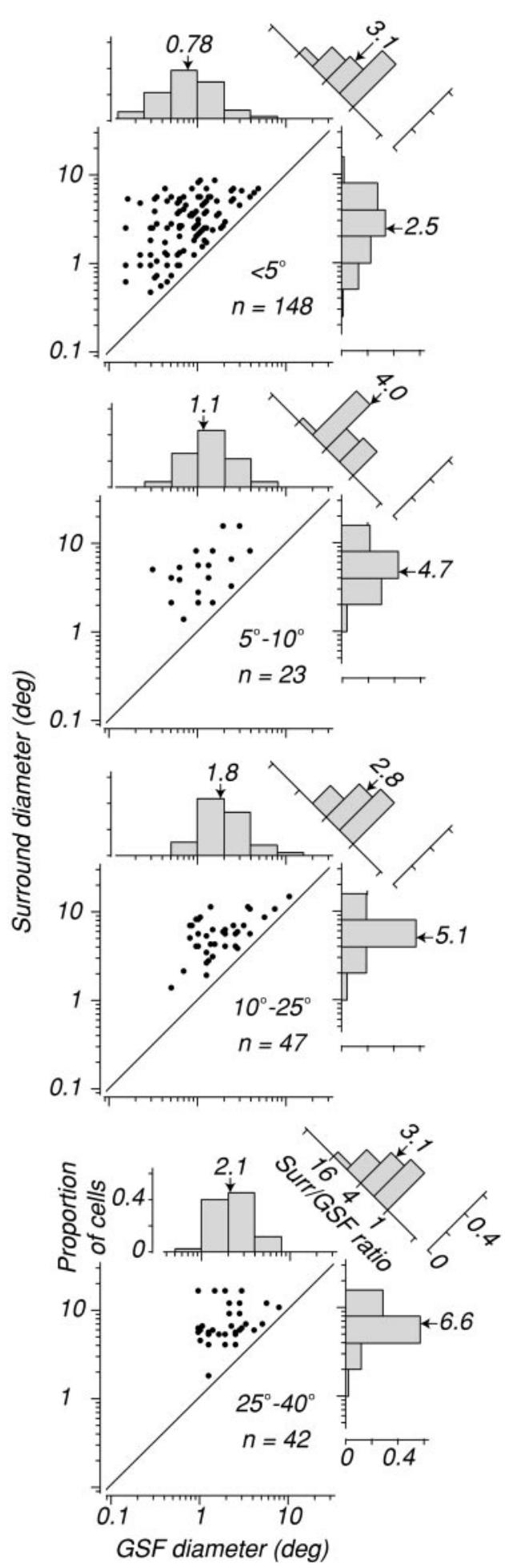

FIG. 2. Center and surround extents extracted from data. Each panel shows the diameter of the surround plotted against the diameter of the center for each of 4 ranges of eccentricities. Marginal histograms show, for each panel, the distribution of GSF diameters (right) and the distribution of surround diameters (top). The ratio of surround to center extent is plotted in the histogram on each diagonal. $\downarrow$, the geometric mean in each distribution. We included neurons for which there was a measurable (at least 10\%) degree of suppression $(n=260)$. Both GSF diameter and surround diameter increased with eccentricity, although the ratio of surround to center diameter (relative surround extent) was fairly constant across eccentricities.

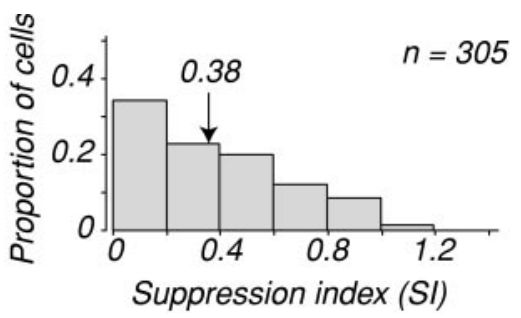

FIG. 3. Distribution of surround suppression for 305 neurons for which there was either suppression or response saturation for the largest patches of grating. Suppression is expressed as a suppression index (SI) - the fractional reduction in the neuron's maximum response. Neurons were suppressed by an average of $38 \%$ of their maximum response by stimuli extending beyond the GSF.

Thus we were unable to systematically differentiate layers with typically vague borders, and so we cannot rule out the possibility of some laminar differences.

On average, neurons were suppressed by $38 \%$ of their maximum firing rate by stimuli extending beyond their classical receptive fields. Figure $3 B$ shows the distribution of suppression indices for all neurons. Only a very small number of neurons ( 2 of 106 with sufficient spontaneous activity) were suppressed below spontaneous firing rate by large patches of grating, even though stimulating the surround often suppressed responses to stimuli within the receptive field. This suggests that inhibitory influences from the surround act by modifying gain, not by subtraction.

When analyzed by lamina, suppression was slightly stronger on average for cells in layer $4 \mathrm{ab}$ and weaker for cells in layer 6 . This trend was not significant based on a $\chi^{2}$ test. Sceniak et al. (2001) found a similar trend in their data set to be significant.

Along with the AMRF and GSF determined from responses to gratings, we also qualitatively assessed the MRF with bars of light. This gave us three independent measures of receptive field extent. We compared qualitative estimates of the MRF with GSF diameters for 217 neurons. The GSF was, on average, about twice the diameter of the MRF. For 162 neurons, AMRFs were on average $47 \%$ larger than GSFs.

For simple cells of a particular preferred spatial frequency, variations in GSF size should be related to variations in spatial frequency selectivity - specifically, cells with large GSFs relative to the period of their preferred frequency are best stimulated when a relatively large number of cycles of the preferred grating fall within their receptive fields. Such cells should have narrow spatial frequency bandwidths, while cells with relatively small GSFs should have broad bandwidths. This trend could be easily seen in our data: the correlation between spatial bandwidth (in octaves) and the ratio of GSF diameter to preferred spatial period was $-0.40(n=123, P \ll 0.001)$. A similar trend would be expected also for orientation bandwidth (cf. De Valois et al. 1985), and we found a similar relationship in our data $(r=-0.35, n=118, P \ll 0.001)$. We conclude that measured variations in GSF diameter correspond to functional summation zones within cortical receptive fields that are related to their spatial selectivity.

More than half of our neurons tested with the annular stimuli $(150 / 255)$ had AMRFs that were larger than their GSFs, meaning that an annular stimulus placed entirely outside the receptive field measured with a patch of grating could still elicit a response. The behavior of these neurons gave us an important 


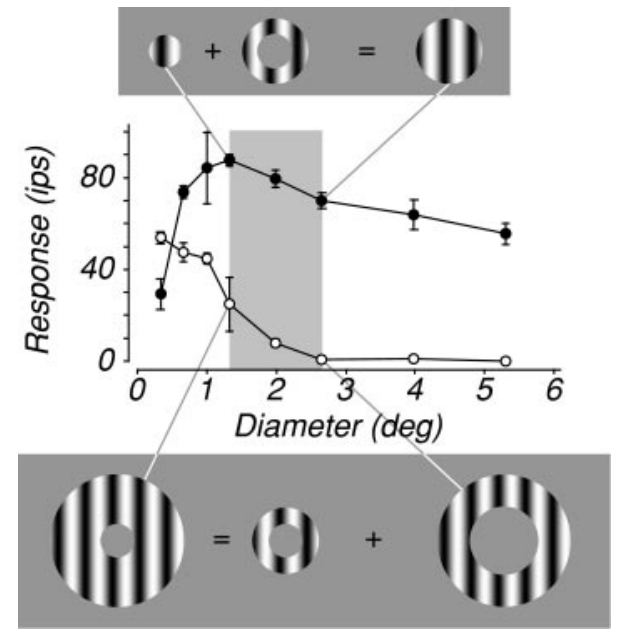

FIG. 4. Certain regions of the receptive field yield different responses depending on stimulus context. $\bullet$, responses to a circular patch of grating; $\bigcirc$, responses to an annular patch of grating. Shading indicates the area outside the GSF from which an annular stimulus still elicited a response. Stimulation of this shaded area yielded suppression when the center was being stimulated by a grating with a diameter of $1.3^{\circ}$ and excitation in the presence of an annular patch of grating with an inner diameter of $2.7^{\circ}$. This suggests that the excitatory and inhibitory influences of the receptive field are independently regulated, their balance determining the overall effect of stimulation.

clue about the relationship between center and surround mechanisms. Consider the example cell whose data are shown in Fig. 4. Responses to circular patches of grating are plotted as 0 and responses to annuli are plotted as $\bigcirc$; the shaded area represents the difference between the two estimates of receptive field extent. For this neuron, the GSF was $1.3^{\circ}$ in diameter, meaning that increases in outer diameter beyond this value caused response to decrease. However, the response to an annular grating began to increase when the inner diameter was made smaller than $2.7^{\circ}$. Thus the region of the receptive field covered by an annulus whose inner and outer diameters were 1.3 and $2.7^{\circ} \mathrm{had}$ an influence on response that depended on the pattern of stimulation of other parts of the receptive field. The schematics at the top and bottom of Fig. 4 make this explicit by showing the effect of this annulus on response. When added to the optimal circular patch of grating, the annulus caused a $20 \%$ reduction in response, but when added to the ineffective annular grating, the same annulus increased the neuron's firing rate to about $30 \%$ of its maximum. We interpret this to mean that in the region defined by this annulus, the neuron's response depended on a combination of influences from the excitatory center mechanism and the suppressive surround. The balance between the excitatory and inhibitory regions determined whether a stimulus would excite or suppress.

We conjecture that center and surround responses arise from independent mechanisms, the gains of which are independently regulated. A stimulus in the center reduces center sensitivity, allowing surround suppression to dominate in the transitional annulus. Similarly, a surround stimulus reduces surround sensitivity, allowing the center to dominate in the transitional annulus. This explains why adding the annulus to the center causes suppression while adding it to the surround causes excitation and suggests that it might be fruitful to try to explain other complex features of responses with a model in which the sensitivity of the center and surround mechanisms are independently regulated. Before building such a model, however, it is necessary to know the form of the interaction between center and surround signals.

\section{Contrast response of the center and surround}

Given independent center and surround mechanisms, suppression from the surround will manifest itself in the neuron's contrast response. Suppression might be either divisive or subtractive, requiring responses at different stimulus contrasts to differentiate the two. Characterizing changes in a neuron's contrast response will tell us whether the influence from the surround should be modeled as a divisive or subtractive suppression.

Figure $5 A$ shows three ways in which a neuron's contrast response might be changed by stimulating the receptive field surround. A horizontal shift in the neuron's contrast response curve represents a change in the neuron's response with stimulus contrast-a change in contrast gain. Changing contrast gain does not typically affect a neuron's maximum firing rate but effectively scales contrast for the neuron. A vertical scaling of the curve represents a change in response dependent on the neuron's firing rate- a change in response gain. This gain change does not alter the range of contrasts to which a neuron responds but simply scales responses at all contrasts. Changes in both contrast gain and response gain are divisive forms of suppression. A third possibility is subtraction in combination with a threshold that reduces responses the same amount at all contrasts. To determine which of these three forms of suppression best characterized surround influences, we considered three models. The first model accounts for surround suppression through a divisive change in the neuron's response gain. This response gain model is

$$
R=K\left(c_{\mathrm{s}}\right)\left(\frac{c_{\mathrm{c}}}{\sqrt{\sigma+c_{\mathrm{c}}^{2}}}\right)^{\beta}
$$

in which $R$ is the neuron's response, $K\left(c_{s}\right)$ is the scaling factor dependent on surround contrast, $c_{\mathrm{c}}$ is the center contrast, $\sigma$ sets the neuron's contrast gain, and $\beta$ sets the slope of the neuron's contrast response function in log-linear coordinates. In this model, contrast response is scaled by a single factor $K\left(c_{\mathrm{s}}\right)$ that depends on surround contrast.

The second model also accounts for the surround influence with divisive suppression, but through a change in contrast gain

$$
R=K\left(\frac{c_{\mathrm{c}}}{\sqrt{\sigma\left(c_{\mathrm{s}}\right)+c_{\mathrm{c}}^{2}}}\right)^{\beta}
$$

For the contrast gain model, the response-scaling factor $K$ is fixed, but the contrast gain parameter $\sigma\left(c_{\mathrm{s}}\right)$ depends on surround contrast.

The third model assumes a subtractive influence from the surround

$$
R=\max \left[0, K\left(\frac{c_{\mathrm{c}}}{\sqrt{\sigma+c_{\mathrm{c}}^{2}}}\right)^{\beta}-k_{0}\left(c_{\mathrm{s}}\right)\right]
$$

in which $k_{0}\left(c_{\mathrm{s}}\right)$ is a response offset that depends on surround contrast. The maximum operation simply imposes a floor at 0 on the response. Each of these models is based on the familiar Michaelis-Menten equation, which well describes the contrast/ 
response relationship in visual cortical neurons (Albrecht and Hamilton 1982).

We collected families of contrast response curves from 66 neurons. We presented center and surround gratings at the neuron's preferred orientation, spatial frequency, and temporal frequency. In these and other experiments, we used our measurements of the GSF and AMRF to make conservative choices for the center and surround regions. We always chose center stimuli to be equal to or smaller than the GSF diameter, reasoning that they were thus confined to a region in which the center mechanism was dominant. To be certain that the surround stimulus did not encroach on the center, we always

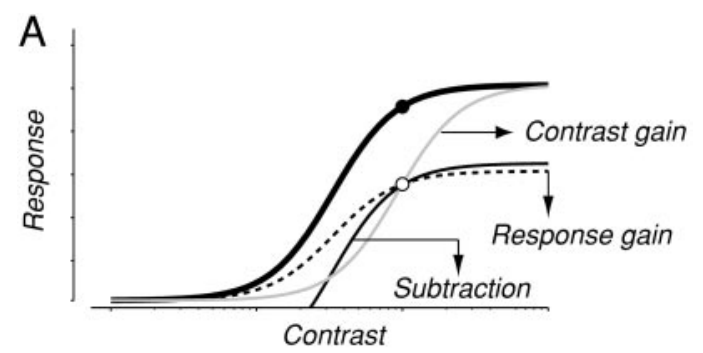

B Cell 1 Cell 2

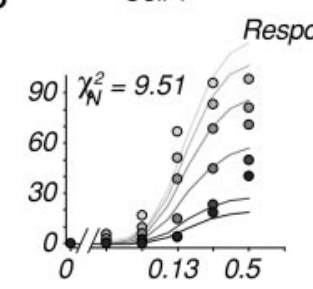

Response gain

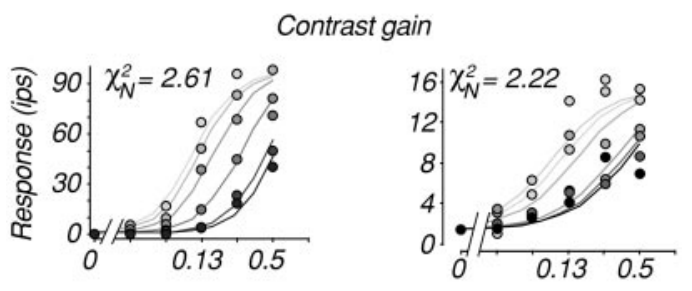

Subtraction
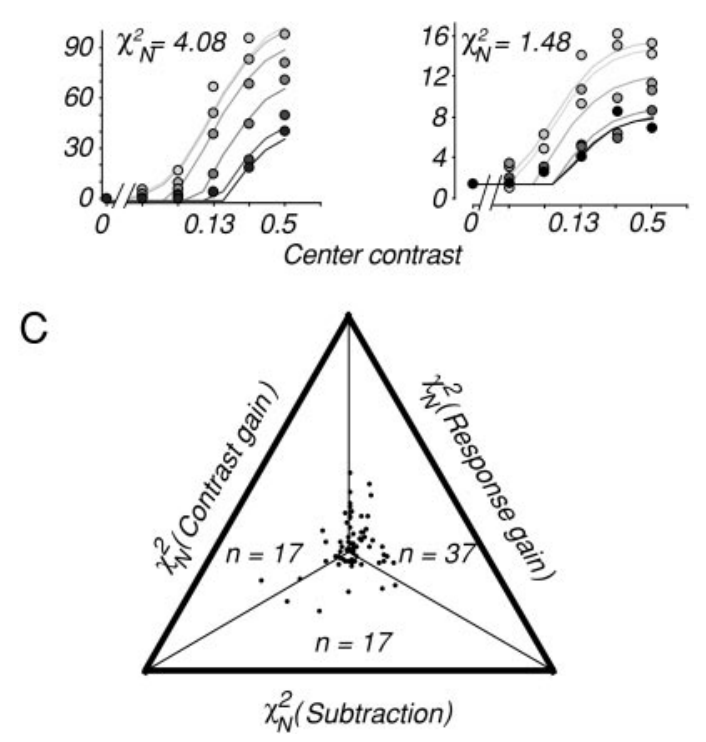

chose surround stimuli to be annuli whose inner diameter was either the AMRF diameter or the GSF diameter, whichever was larger.

We independently varied the contrasts of the center and surround stimuli and for each neuron obtained a family of six contrast response curves. The data (circles) in Fig. $5 B$ are examples of families of contrast response curves recorded from two neurons. Each set of points shows the response of the neuron to increasing center contrast in the presence of a single surround contrast. The shading of the points in each curve represents surround contrast: white for $0 \%$ surround contrast to black for $50 \%$ surround contrast. Responses of a simple cell are shown on the left. For this neuron, an increase in surround contrast caused a rightward shift in the contrast response curve as indicated by the disappearance of response saturation at higher surround contrasts. For the responses of the complex cell shown on the right, there was a change in slope and a loss of response saturation, suggesting that none of the three models would completely account for the effect of increasing surround contrast.

We fit our models to the response magnitudes of our contrast response curve families. Sample fits of each model for two cells are shown in Fig. $5 B$ as solid curves. The top panels show fits to the response gain model, the center panels show fits to the contrast gain model, and the bottom panels show fits to the subtractive model. The shading of each curve indicates surround contrast, with darker shades representing higher contrasts. Each panel gives $\chi_{N}^{2}$ for that model's fit to the responses (see METHODS). The neuron for which the contrast response curve shifted horizontally with surround contrast (left) was best fit by the contrast gain model. On the other hand, the neuron for which responses seemed to scale vertically with surround con-

FIG. 5. Different forms of surround suppression. A: 3 ways in which a neuron's contrast response can change. The bold curve represents a neuron's unmodified response to stimuli at different contrasts. The two thinner solid curves represent 2 divisive forms of suppression. A change in contrast gain (thin gray curve) is a divisive suppression that effectively scales stimulus contrast for the neuron. A change in response gain (dashed curve) is a divisive suppression that scales responses equally at all contrasts. The 3rd type of suppression shown is subtractive (thin black curve), which suppresses the same amount at all contrasts. $B$ : responses and fits for 2 sample cells (left and right) to models accounting for surround suppression either by division (top and middle) or subtraction (bottom). The circles in each panel represent responses to a compound center/surround stimulus in which we varied the contrast of each component grating independently. The abscissa designates the contrast of the center patch of grating, while the shading of each circle denotes the contrast of the surround stimulus with darker shades representing higher contrasts (lightest to darkest circles $=0,3,6,12,25$, and 50\% contrast, respectively). Responses decrease as surround contrast increases (lighter to darker circles). Solid curves are fits of the 3 models. Top panels show fits to the response gain model-modeled contrast responses are vertically scaled versions of one another. Cell 2 (right) was fit best by the response gain model. Middle panels show fits to the contrast gain model-surround contrast shifts the curves horizontally. Cell 1 (left) was best fit by the contrast gain model. Bottom panels show fits to the subtractive model, which performed reasonably for both cells. Inset in each panel is the $\chi^{2}$ error normalized by the degrees of freedom in each model $\left(\chi_{N}^{2}\right)$. $C$ : comparison of $\chi_{N}^{2}$ for 3 models. Axis units are normalized, making the total $\chi_{N}^{2}$ value for all 3 models equal 1 . The distance of a point from each bounding axis represents its $\chi_{N}^{2}$ for the fit to that model. Points in the center of the plot represent data fit equally well by all 3 models. Points near a bounding axis represent cells for which that model performed better than the others. The response gain model performed best, indicated by the number of points nearest that axis. When we took response phase into account for simple cells, the response gain and contrast gain models performed equally well. The subtractive model on average did the poorest job accounting for surround suppression. 
trast (right) was best fit by the response gain model. The subtractive model also provided good fits in both cases, but in neither case was the best. Although the models provided good fits qualitatively, the $\chi_{N}^{2}$ were often well above 1 (e.g., for contrast gain in cell 1). This was often due to model deviations from the large number of small responses at low contrast; response variances were proportionately low and thus provided greater penalties when fitting.

Each model provided adequate fits to responses from most neurons (mean $\chi_{N}^{2}=2.15$ for response gain, 2.24 for contrast gain, and 2.38 for subtraction). We compared the three models on the basis of their $\chi_{N}^{2}$ values, which accounted for differences in the number of degrees of freedom. We made a three-way relative comparison (Fig. 5C) by plotting a point in a triangular space in which the distances of a point from the three edges were in proportion to the $\chi_{N}^{2}$ values for the three models indicated by the edge labels. If all three values of $\chi_{N}^{2}$ were equal for a neuron, its point would lie at the center of the triangle. If the $\chi_{N}^{2}$ for only one of the fits was zero, the point would lie on the edge corresponding to that fit. The point for each cell therefore lies in the triangle closest to the edge corresponding to the model that provides the best fit for its data. The distribution of points favors the response gain model, while fewer than $25 \%$ of neurons were best described by a subtractive change in response. When we fit the simple cell subpopulation of our sample (23/66 units) to forms of the models that accounted for the effects of contrast gain on response phase (Carandini et al. 1997b), the numbers of units better described by the contrast gain model (10/23) and by the response gain model (13/23) were roughly equal.

The subtractive model, when compared with the two divisive forms of suppression, performed best in less than a quarter of the neurons. In addition, fits to a model which used both contrast gain and response gain to account for surround suppression produced an improvement in $\chi_{N}^{2}($ mean $=1.82)$, suggesting that using both of these forms of divisive gain provides a better description of the data than either one alone. Because previous results have suggested that suppression is mediated by divisive changes in gain (DeAngelis et al. 1994; Sengpiel et al. 1998; Somers et al. 1998), we conclude that the influence of the surround is best considered as some form of divisive or modulatory suppression. We considered two different forms of divisive interaction, contrast gain and response gain. Our laboratory has previously shown that contrast gain models account well for suppressive effects within the classical receptive field (Carandini et al. 1997b). In the case of surround suppression, however, our analysis suggests that the response gain model provides a better description. For the purposes of the remaining work in this paper, the two models are equivalent; more refined experiments are needed to decide which description is more accurate.

\section{Stability and independence of center and surround mechanisms}

Recall that stimulus context appears to differentially set the gains of the center and surround (Fig. 4) and that we have just concluded that the surround acts through some divisive form of suppression. We now describe and test a receptive field model that assumes independent center and surround mechanisms in which the surround influences responses through a divisive

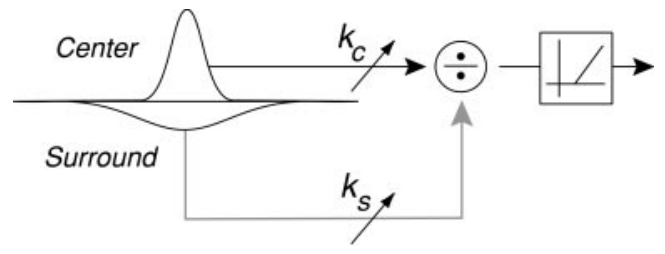

FIG. 6. The ratio of Gaussians (RoG) model. We constructed the RoG model from independent and spatially stable center and surround components. We modeled each component as a Gaussian envelope of sensitivity incorporating the spatiotemporal tuning characteristics of a neuron. Each component had an independently controlled gain, and the surround affected the model cell's response through divisive suppression. The model is instantiated in Eq. 9 through 11 .

gain control. This model is intended to provide a simple explanation of changes in receptive field size by using mechanisms with spatially constant dimensions.

Based on the general shape of diameter tuning curves, we modeled the sensitivity distribution of each mechanism with a one-dimensional Gaussian envelope of sensitivity. Integration of this one-dimensional Gaussian corresponds to integrating a two-dimensional envelope of the form

$$
\frac{\exp \left(\frac{-r^{2}}{2 \sigma^{2}}\right)}{r}
$$

where $r$ is radius and $\sigma$ is the SD of the Gaussian envelope. This envelope determined the spatial extent of the receptive field mechanisms. It is important to understand that the Gaussian envelopes do not describe the spatial weighting function of the receptive field but only the envelope of that function. So for a linear approximation to a simple cell, the center envelope would correspond to the Gaussian envelope of a Gabor filter (Movshon et al. 1978a). For a complex cell, the center envelope would correspond roughly to the receptive field map obtained from a line weighting function (Movshon et al. 1978b). Figure 6 shows a schematic of this model. The left portion of the model shows the overlapping one-dimensional sensitivity envelopes. We calculated the activity of each mechanism by taking the integral under each Gaussian envelope covered by a stimulus placed over their common centers. The model divides the output of the center mechanism by the output of the surround mechanism; the gain for each mechanism is independently controlled by the gains $k_{\mathrm{c}}$ and $k_{\mathrm{s}}$. This divisive interaction of center and surround mechanisms formed a ratio of Gaussians (RoG). To model responses to circular patches of grating we take

$$
R(x)=\frac{k_{\mathrm{c}} L_{\mathrm{c}}(x)}{1+k_{\mathrm{s}} L_{\mathrm{s}}(x)}
$$

where

$$
L_{\mathrm{c}}(x)=\left(\frac{2}{\sqrt{\pi}} \int_{0}^{x} e^{-\left(y / w_{\mathrm{c}}\right)^{2}} \mathrm{~d} y\right)^{2}
$$

and

$$
L_{\mathrm{s}}(x)=\left(\frac{2}{\sqrt{\pi}} \int_{0}^{x} e^{-\left(y / w_{\mathrm{s}}\right)} \mathrm{d} y\right)^{2}
$$

in which $x$ is stimulus diameter, $k_{\mathrm{c}}$ and $k_{\mathrm{s}}$ are the gains of the center and surround mechanisms, and $L_{\mathrm{c}}$ and $L_{\mathrm{s}}$ are the 
summed squared activities of the center and surround mechanisms, respectively. The spatial extents of the center and surround components were represented by $w_{\mathrm{c}}$ and $w_{\mathrm{s}}$. We always constrained $w_{\mathrm{c}}<w_{\mathrm{s}}$.

This model implements a general divisive normalization, so Eq. 9 differs from previously considered divisive models (Eqs. 5 and 6 ), which capture particular kinds of divisive normalization (changes in response gain and contrast gain). Rather than explicitly describe the neuron's contrast/response relationship, we devised a new model according to a more generalized concept of divisive normalization as illustrated in Fig. 6. This generalized RoG model is similar to the one used by Chen et al. (2001) to model the effect of patches of grating flanking the CRF on a neuron's contrast response.

\section{Changes in receptive field structure with contrast}

Sceniak et al. (1999) and Kapadia et al. (1999) have recently demonstrated that the spatial extent of the receptive field appears to change with stimulus contrast: at lower contrasts, neurons prefer larger stimuli. Although at first it might appear that the underlying mechanisms must grow as contrast decreases (Sceniak et al. 1999), we decided to use our model to test whether simply changing the gains of fixed center and surround mechanisms could account for changes in measured receptive field extent with contrast. Figure 7 illustrates the manner in which receptive field extent can change assuming spatially stable center and surround mechanisms. The center and surround mechanisms (gray lines) are identical in each panel save for their gains, which are represented by the thickness of the lines. We calculated the resulting apparent receptive field (solid black line) by dividing the center mechanism by the surround mechanism, and imposing a response threshold (dashed black line). The boundaries of the resulting receptive field (shaded area) are designated by the dotted vertical lines, and the width of the receptive field is noted in each panel along with the relative gain of the surround mechanism. For strong surround gains, the resulting receptive field is small. As surround gain decreases, more of the receptive field is uncovered, resulting in an expansion of the measured receptive field. This receptive field expansion is purely a function of the changing balance between center and surround gains.

We measured grating diameter tuning curves at different stimulus contrasts for 79 neurons in primary visual cortex. As stimulus contrast decreased, not only did responses decrease, but also the shape of the diameter tuning curves changed, resulting in a preference for larger stimuli (data in Fig. 8, $A-C$ ). For stimuli at the lowest contrast (6\%, lightest points in Fig. 8, $A-C)$, GSF diameters were on average about 2.5 times those measured at high contrast (darkest points), confirming the results of Sceniak et al. (1999) and Kapadia et al. (1999).

These data demonstrate that the area of summation of V1 cells changes with stimulus contrast. To determine whether this change in spatial summation required a change in the spatial extents of the underlying mechanisms, we fit three forms of our ratio of Gaussians model to families of diameter tuning curves showing receptive field expansion. In the first form of the model, we permitted only the central gain parameter, $k_{\mathrm{c}}$, to vary with contrast. We designated this the uniform model because changing only the center gain produces a family of
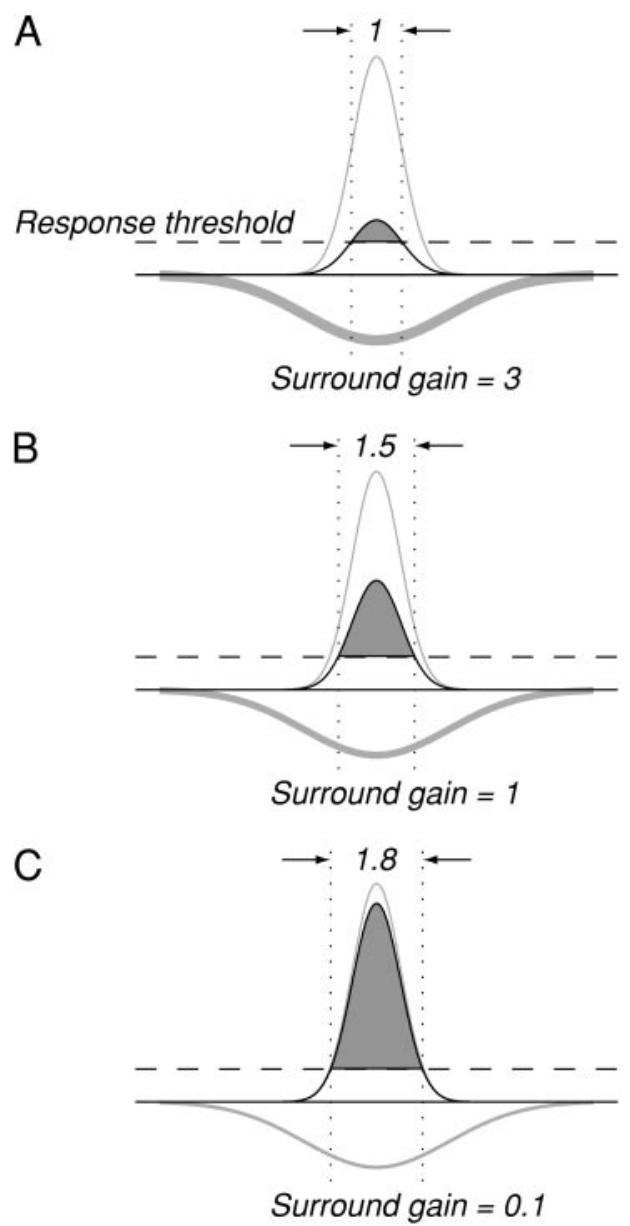

FIG. 7. Stable center and surround mechanisms can account for changes in receptive field size. Each panel shows a schematic representation of the Gaussian center and surround components in our RoG model (solid gray curves). Line thickness represents the relative gains of each component. The gain of the center component is held constant for each panel, but the gain of the surround changes from $3(A)$ to $1(B)$ to $0.1(C)$. We calculated the resulting receptive field profile (solid black curve) by dividing the center mechanism by the weighted surround mechanism as in the RoG model. After imposing a response threshold (dashed line), we measured the width of the receptive field (shaded area). As surround gain decreased, the size of the resulting receptive field increased from 1 when surround gain was 3 , to 1.8 when surround gain was virtually absent. The weakening of the surround allows the fringes of the receptive field to become responsive, increasing its apparent size.

curves that are scaled versions of each other. In the second form of the model, the surround gain parameter, $k_{\mathrm{s}}$, was also permitted to vary with stimulus contrast while the widths of the sensitivity envelopes were held constant. This was the gain model, as it permitted center and surround gains to be independently regulated. In the final form of the model, we additionally permitted the width of the center sensitivity envelope $\left(w_{\mathrm{c}}\right)$ to change with contrast. We called this the size model, as it allowed the changing extent of the center mechanism to help explain receptive field expansion.

The fits of each version of the model to the responses of the sample neuron are also plotted in Fig. 8, A-C. Fits are plotted as solid curves in each panel, the shade of each curve corresponding to stimulus contrast. The top panel shows the fit to the uniform model, the middle panel shows fits to the gain model, and the third panel shows fits to the size model. 
A

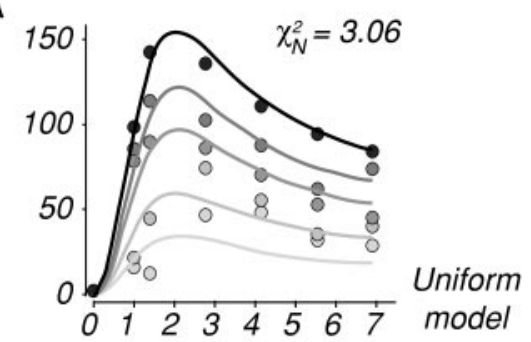

B

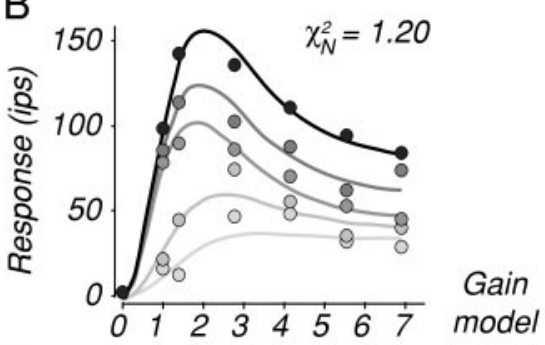

C
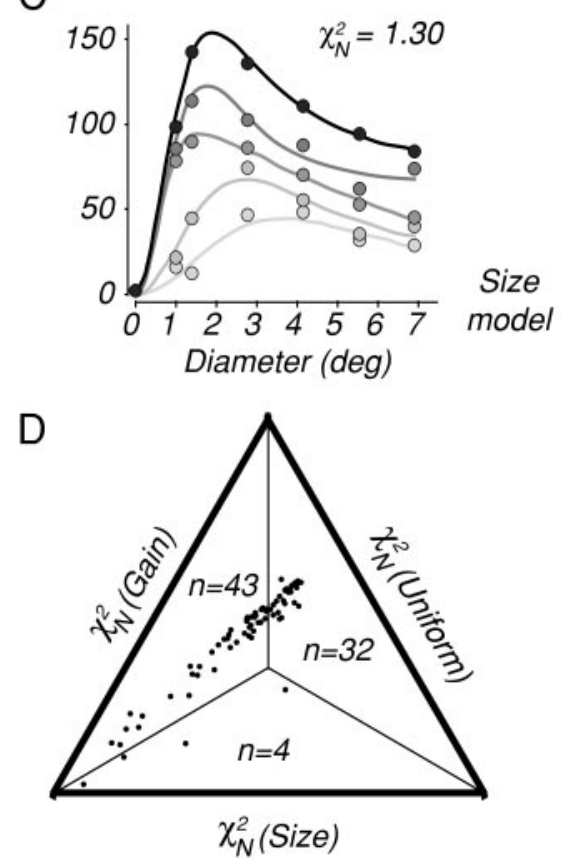

FIG. 8. Receptive field size changes with stimulus contrast. $A-C$ : responses of a single cell (filled circles) are duplicated in each panel. We have plotted response as a function of stimulus diameter, the shading of each circle representing stimulus contrast (darker circles indicate higher contrast: 6, 13, 25, 50, and 100\% contrast, respectively). As stimulus contrast decreased, responses decreased more for small stimuli than for large, resulting in a shift in diameter tuning. Solid curves show the fits for each of 3 versions of the RoG model: uniform, gain, and size. The shading of each line indicates stimulus contrast. The uniform model only varied the gain of the center component in the RoG model, whereas both center and surround gains varied in the gain model. In the size model, the extent of the center component varied with stimulus contrast as well. The normalized $\chi^{2}$ error for each fit $\left(\chi_{N}^{2}\right)$ is shown in each panel. Both the gain and size models captured the change in receptive field size with contrast, but $\chi_{N}^{2}$ was smaller for the gain model, indicating that changing the width of the center mechanism yielded no substantial improvement in fit quality. $C$ : comparison of $\chi_{N}^{2}$ among the 3 models for 79 neurons (see Fig. 5 for explanation of axes). Although each model often provided fits of similar quality (points near the center), the majority of points are closer to the axis for the gain model, indicating that the model with spatially stable center and surround components accounted for the data best. Permitting mechanism width to vary in the size model did not improve fit quality when taking into account the number of free parameters in each model.
When only center gain was allowed to vary (Fig. 8A), the curves produced by the uniform model were vertically scaled versions of each other. As expected, the uniform model was unable to account for the shift in optimal stimulus diameter with changes in contrast. When surround gain was also permitted to vary in the gain model (Fig. $8 B$ ), the curves accounted well for the reduction in response and for the shift in GSF diameter. When additionally the extent of the center spatial envelope was permitted to vary in the size model (Fig. $8 C$ ), fit quality again visibly improved.

Mean $\chi_{N}^{2}$ were 0.99 for the uniform model, 0.86 for the gain model, and 1.14 for the size model. Thus all three forms of the model provided acceptable accounts of most of the data. The mean $\chi_{N}^{2}$ was lower for the gain model than for the uniform model, indicating an improvement in fit quality, even taking into account the addition of the extra varying parameter. However, when the center mechanism width was allowed to vary as well in the size model, there was an increase in the $\chi_{N}^{2}$, implying that although the absolute error decreased when adding this parameter to the model, this reduction in error did not warrant the extra parameter.

Figure $8 D$ shows a three-way comparison of $\chi_{N}^{2}$ among models (see Fig. $5 C$ ). The points fall predominantly in the sector for the gain model in which center and surround gains only $\left(k_{\mathrm{c}}\right.$ and $\left.k_{\mathrm{s}}\right)$ were permitted to vary with contrast, indicating that this model provided the best overall description of the data. Permitting the center mechanism width to also vary with contrast in the size model did not provide better fits than the gain model. Thus the model providing the most parsimonious explanation of the data was the gain model, in which changes in receptive field extent were accounted for by independent changes in gain of spatially fixed center and surround mechanisms. The gain parameters in this model accounted for the changes in mechanism activity with contrast and thus reflect each mechanism's sensitivity to contrast. The way that the balance of the gains in the model creates changes in receptive field size is the one schematized in Fig. 7. At high contrasts, the surround is relatively strong and suppresses weak responses from the flanks of the center mechanism. At low contrasts the surround is relatively weak, and this suppression is relaxed, allowing more of the center to be seen.

To document this point, Fig. $9 A$ shows how the average center signal $\left(k_{\mathrm{c}}\right)$ across neurons develops with increasing contrast in the gain model. Because contrast was not explicit in our model, it has been absorbed into the gain parameters that thus capture the way that signals depend on contrast. We plot means of signal values normalized by their maximum for each neuron. At low contrasts, the signal of the center component was weak, and it increased with contrast. Figure $9 B$ shows the average development across neurons of the surround signal $\left(k_{\mathrm{s}}\right)$ with contrast. Surround signals were also weak at low contrasts and also increased for high contrast stimuli. The key to understanding the changing role of the two mechanisms with contrast is to visualize the relative sensitivity of each mechanism to contrast. We gauged the relative effect of surround suppression in a manner analogous to the measurement of suppression in diameter tuning curves by measuring the suppressive influence relative to the excitatory influence. Without suppression, the overall response to large (infinite) diameter stimuli is proportional to $k_{\mathrm{c}}$. With divisive suppression, the response was 

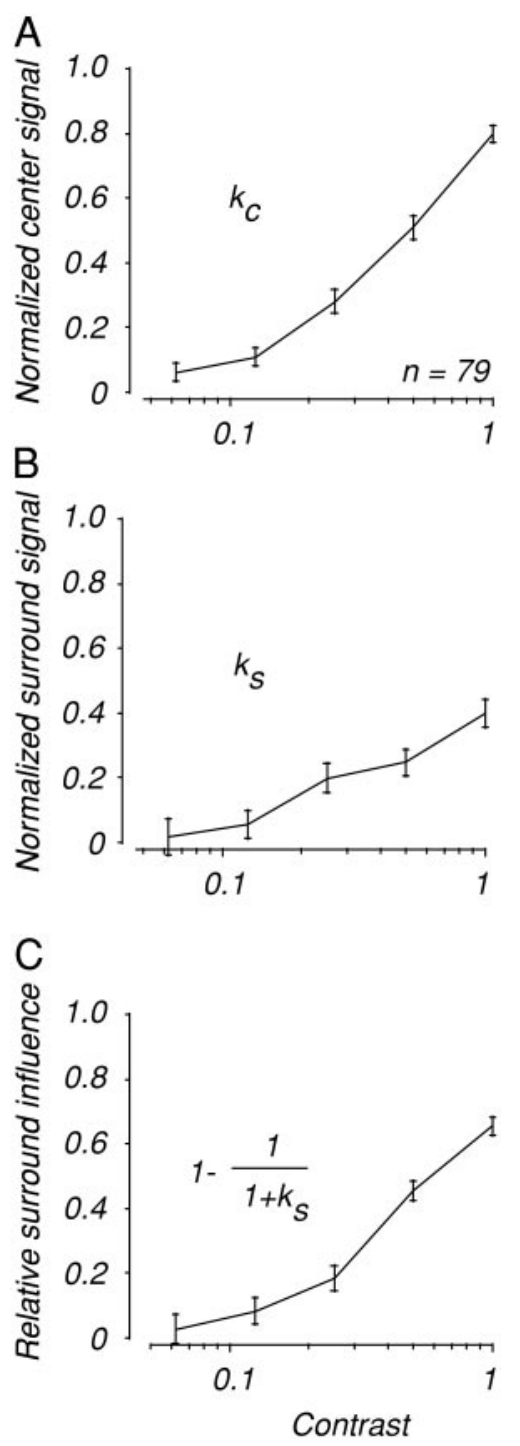

FIG. 9. Changes in RoG model parameters with contrast. We extracted center and surround gain parameters from fits to the gain RoG model for 79 cells. Because contrast was not explicit in our model, it has been absorbed into the gain parameters, labeled center and surround signals in the figure. Thus the center and surround gain parameters are expected to increase with contrast. What is interesting is the comparison between the two. For each neuron, we normalized the gain parameters to their maximum value, and averaged these normalized quantities for all neurons. $A$ and $B$ : center signal increases with contrast more than surround signal. $C$ : we expressed the asymptotic influence of the surround as the expected reduction in model activity (see $E q$. 12). The relative influence of the surround increases with contrast, suggesting that the surround is less sensitive than the center at lower contrasts.

proportional to $k_{\mathrm{c}} /\left(1+k_{\mathrm{s}}\right)$. Expressing suppression as a fractional reduction in response, we obtain

$$
S=1-\frac{1}{1+k_{\mathrm{s}}}
$$

where $S$ is the suppression value. Because we constrained $k_{\mathrm{s}}>$ 0 , it must be that $0<S<1$. Figure $9 C$ shows that relative surround gain $S$ fell from its high contrast value of about 0.65 to near zero at low contrast. We conclude that the influence of the surround was, on average, drastically weaker at lower contrasts than at high, and that this difference in the contrast gain characteristics of center and surround mechanisms is what causes the apparent change in receptive field size with contrast.

\section{Changes in receptive field structure with adaptation}

Encouraged by our finding that changes in spatial summation with stimulus contrast are accounted for by changes only in contrast gain, we turned our attention to the question of how other changes in gain might affect receptive field structure. Cortical cell responses fall during prolonged stimulation with high contrast targets, because of a change of contrast gain (Sclar et al. 1989; Vautin and Berkley 1977). We earlier speculated that the center and surround gains might be independently controlled by adaptation (Fig. 4). We now test this conjecture by asking whether the gain model just described can be used to account for the effects of adaptation over the 1.5- to 6-s duration of each of our stimulus presentations.

Figure 10 shows an analysis of the time course of the responses of a complex cell to patches of grating of different diameters. Mean responses are plotted in the left panel as a grating diameter tuning curve, and the time histograms for each response are plotted in the right panel. In the diameter tuning curve, the responses to stimuli with diameters of 0.69 and $2.78^{\circ}$ $(A$ and $B)$ are highlighted with circles. Mean response rates to these two stimuli were similar (13.8 and 14.1 spikes/s, respectively), but the histograms show the responses to have very different time courses (right, indicated by the $\downarrow, A$ and $B$ ). In the first $2,000 \mathrm{~ms}$, the response to the smaller diameter stimulus $(A)$ was greater than the response to the larger stimulus $(B)$, and this relationship reversed in the second $2,000 \mathrm{~ms}$, when the response to the smaller stimulus diminished.

To track the time course of responses, we divided spike trains into fixed-duration epochs. We determined the duration of the epoch by first examining the distribution of cycle drift periods for all cells. We chose a canonical epoch duration of $640 \mathrm{~ms}$, which was short enough to provide reasonable temporal resolution of response trends over time, yet long enough to usually provide reliable response rates within a single epoch. Figure 10, right, shows a series of alternating shaded and unshaded areas, representing the time windows used. Each time window has been given a label $(t 1-t 7)$ for later reference.

We used spikes beginning $150 \mathrm{~ms}$ after the stimulus appeared. This offset accounted for response latencies and minimized the influence of response transients in the first time window. Stimulus duration varied from $1.5 \mathrm{~s}$ to over $5 \mathrm{~s}$, yielding from two to eight response epochs. Within each time epoch, we calculated the neuron's response to gratings of different diameters. We organized these responses into families of patch diameter tuning curves with one curve for each time epoch. For this analysis, we used data from 208 units that were studied with suitable stimulus epochs and which had measurable suppression (greater than 10\%).

Temporal partitioning of responses yielded a family of grating diameter tuning curves for each neuron. A family of such curves is shown in Fig. $11 A$ for a single example cell. Stimulus duration was about $5 \mathrm{~s}$, so temporal partitioning at $640 \mathrm{~ms}$ resulted in a family of seven diameter-tuning curves for this cell. The diameter-tuning curve for the earliest time window (t1) is plotted as the topmost curve (shaded line) in Fig. 11A. The shading of the points on each curve represents the relative time of the responses, with lighter points denoting later times. 

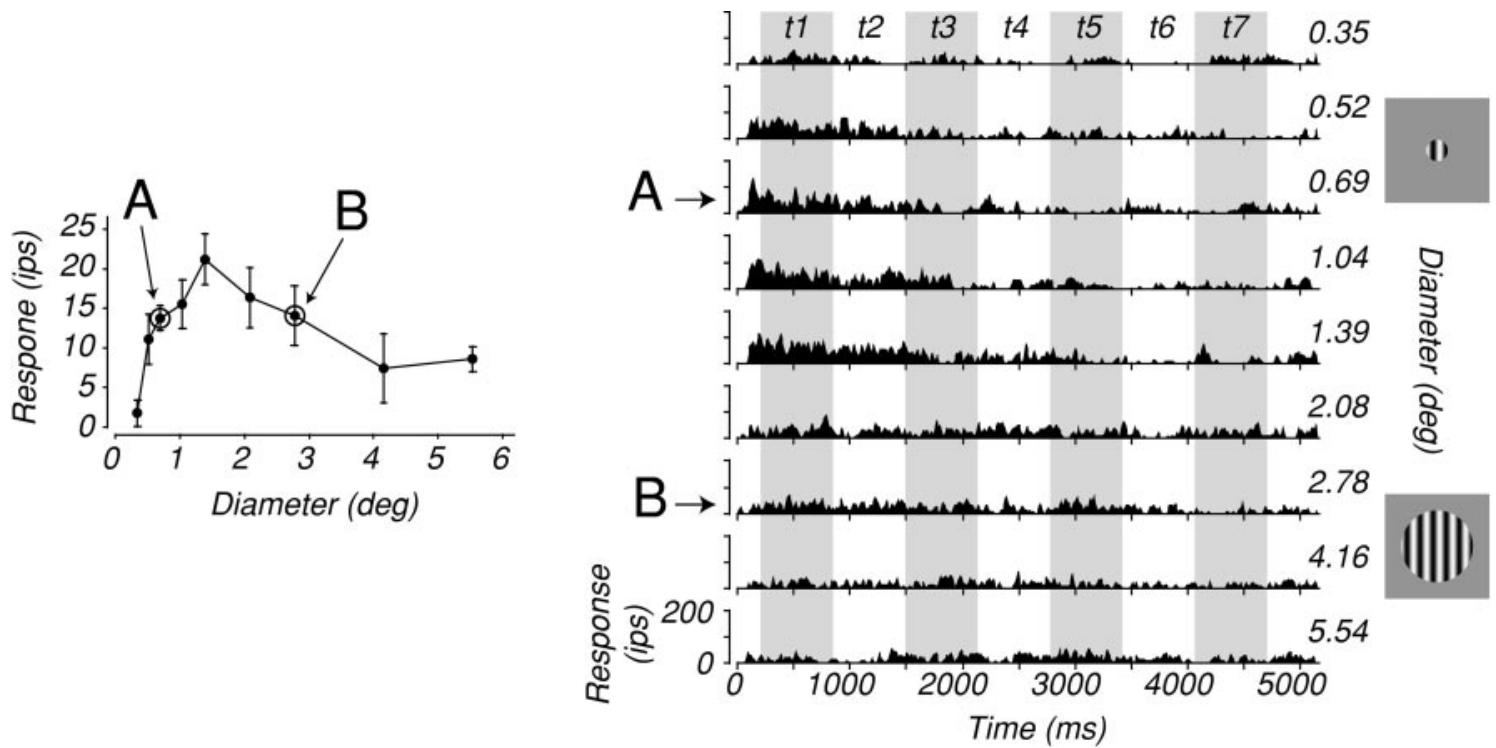

FIG. 10. Time course of responses depends on stimulus diameter. Responses to patches of grating with different diameters are shown for a complex cell. Left: we have plotted response as a function of stimulus diameter. The time course of each response in the left panel is shown in the panel on the right, with responses to smaller stimuli at the top. Responses with similar mean firing rates $(A$ and $B$, left) had very different time courses $(A$ and $B$, right). The response to the smaller stimulus $(A)$ started strong, then faded over $2 \mathrm{~s}$. The response to the larger stimulus was initially lower than the response to the smaller stimulus, but maintained its response rate for $4 \mathrm{~s}$. This resulted in a preference for the smaller stimulus at the beginning of the response and for the larger stimulus at the end of the response.

The neuron's baseline is plotted as the first point in each curve, at $0^{\circ}$ diameter and has been carried across each curve as a dashed line. Because curves from subsequent time windows have been shifted down for visibility, they can be compared by using the dashed baseline as a reference.

As time progressed, responses decreased as the neuron adapted to the stimulus. There were, however, different de-

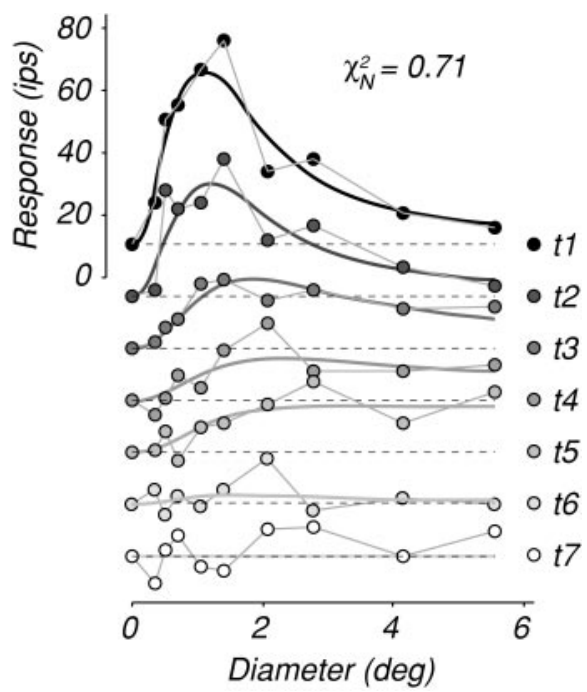

FIG. 11. The RoG model accounts for differential adaptation of the center and surround. A series of grating diameter tuning curves is shown for the complex cell in Fig. 10. Each curve shows the neuron's response as a function of stimulus diameter taken during a different time epoch, indicated by the shading of the circles (lighter shades denote later times). The dashed line indicates the baseline for each curve and serves as a basis for comparison for later curves, which have been shifted down for visibility. Superimposed on the data are the fits from the gain form of the RoG model. The normalized error $\chi_{N}^{2}$ shows that the model's prediction provided a good fit to the data, capturing the expansion of the receptive field over time, and suggests that this expansion is due to differential changes in gain of the center and surround. grees of adaptation to large and small stimuli, resulting in a change in shape of the diameter tuning curve with time. At later times, the neuron preferred larger stimuli. This change in measured receptive field diameter was characteristic of our population of neurons. We calculated GSF diameters for each time window and compared them to the diameter in the earliest window. As time increased, the size of the measured receptive field increased as well, eventually (at around 3 s) becoming about 1.8 times as large as the estimate for the earliest responses. Adaptation produced a change in receptive field size that is qualitatively similar to the change produced by reducing stimulus contrast. We wondered whether the changes could be accounted for in the same way.

For the 208 neurons included in this analysis, we fit the gain model to time-varying families of grating diameter tuning curves in the manner previously described. The smooth curves in Fig. 11A show the fits of the model. Fits for these data were qualitatively similar to those of the contrast-varying curves (Fig. $8 B$ ). The shading of the curves denotes time, with lighter shades representing later times. For this neuron the gain model provided a good fit to the data, capturing the change in receptive field extent over time.

Over our sample of neurons, the geometric mean $\chi_{N}^{2}$ was 0.99 for the gain model, indicating that changes in gain of spatially stable center and surround mechanisms accounted well for the change in receptive field extent due to differential center and surround adaptation. For completeness we compared the gain model to the other two models (uniform and size) and again the gain model performed best (compared with $\chi_{N}^{2}=1.13$ for the uniform model, and 1.06 for the size model).

Viewing the change in receptive field extent with adaptation as a change in the balance of center and surround gains in the context of our model permits us to directly observe how these gain parameters change over time. Figure 12 shows these 

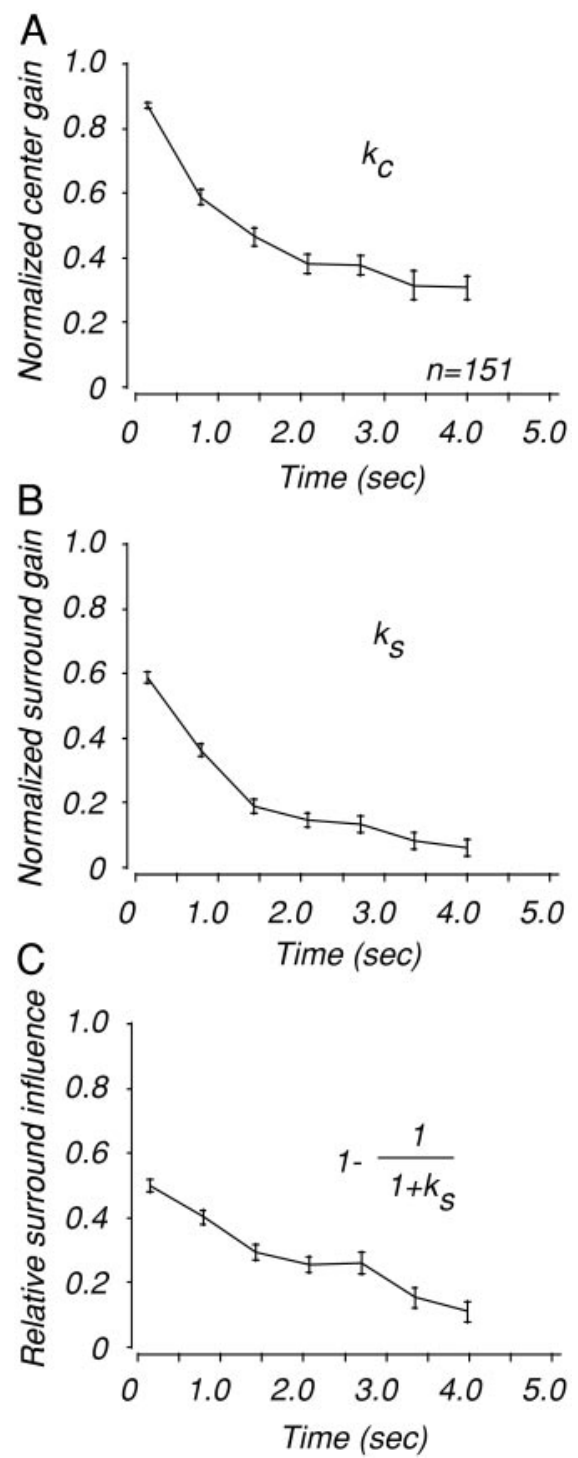

FIG. 12. Changes in RoG model parameters with adaptation. We extracted center and surround gains from fits to the gain RoG model for 208 cells. Fewer data were available at later times due to variable stimulus duration, but each point is the average of data from at least 151 neurons. Parameters were normalized as in Fig. 9. $A$ and $B$ : both center and surround gains decreased over time, corresponding to adaptation of these mechanisms, but the reduction in surround gain was more drastic. $C$ : the asymptotic suppressive influence of the surround (see Eq. 12) decreased with adaptation, suggesting that the surround adapts more than the center and changes the balance of gain in favor of the center, resulting in the appearance of larger receptive fields.

changes, using the same measures and conventions as Fig. 9. The gain values are normalized for each neuron. Figure $12 \mathrm{~A}$ shows the change in the average center gain parameter $\left(k_{\mathrm{c}}\right)$. Center gain was initially high and decreased over the course of $3-4 \mathrm{~s}$ to its final value. Figure $12 B$ shows mean surround gain $\left(k_{\mathrm{s}}\right)$ over time. Surround gain also began at its highest value and then decreased, falling nearly to zero after about $4 \mathrm{~s}$. As was the case with contrast, the change in surround gain was more dramatic than the change in center gain. Figure $12 \mathrm{C}$ shows how the relative surround gain changed over time (see $E q .12)$. The relative strength of the surround decreased sharply over time. Just as the apparent expansion of the receptive field can be attributed to a reduction in surround strength at low contrasts, so also can the apparent expansion of the receptive field with time be attributed to a reduction in surround strength with adaptation. The difference between center and surround in their contrast sensitivity and adaptation characteristics in our model accounts for a wide range of behavior and also suggests that signals in the center and surround might have different origins.

\section{I S C U S S I O N}

\section{How big are V1 receptive fields?}

Researchers often use the MRF estimate of receptive field extent. We compared our initial qualitative estimates of MRF size and quantitative estimates of receptive field extent obtained from grating diameter tuning curves and found that the MRF consistently underestimated our quantitative GSFs and annular minimum response fields AMRFs. Walker et al. (2000) showed that MRF measurements of receptive field extent in cat provide smaller estimates than measurements obtained with patches of grating, showing mean receptive field diameters less than half as large for the MRF as for the GSF. We observed the same qualitative trend in monkey. The small stimuli used in MRF assessment may not provide adequate stimulation to the insensitive fringes of the receptive field. Although stimulation of these areas alone provides only subthreshold stimulation to the neuron, they may facilitate responses elicited by centrally placed stimuli. Facilitation from areas outside the MRF has been reported by a number of groups (e.g., Kapadia et al. 1995). Because these effects all seem to come from zones close to the edge of the MRF, our measurements using summation techniques almost certainly include these facilitatory areas in estimates of receptive field extent. This means that by our measurement and analysis, the regions that others claim to be outside the classical receptive field in fact lie entirely within a seamless region of excitatory effect whose fringes simply have lower sensitivity than its center. This does not of course call into question the finding that these fringe regions of the receptive field provide facilitation. It does, however, make the results less remarkable than they first appeared to be because they simply reflect a rather conventional form of subthreshold summation rather than any more elaborate system for feature linking across large cortical distances. This may also explain why there have been conflicting reports in the literature about the prevalence of facilitatory influences from outside the classical receptive field (Kapadia et al. 1995; Maffei and Fiorentini 1976; Nelson and Frost 1985). A conservative definition of the receptive field such as the MRF leaves plenty of opportunity for facilitatory influences to be found "outside" the receptive field, while a definition based on summation is likely to leave only suppressive influences outside the defined center region.

In the subset of neurons for which we examined the effect of contrast on receptive field size, the mean GSF diameter was $1.3^{\circ}$ for eccentricities less than $5^{\circ}$. The width of the center mechanism in our model agreed with our measurement of GSF. Taking the width of the center mechanism to be $2 \sigma$ of the Gaussian envelope (i.e., $2 w_{c}$ ), center width averaged $1.4^{\circ}$ for receptive fields with small eccentricities (less than $5^{\circ}$ ), and exhibited a strong and significant correlation with GSF diam- 
eter $(r=0.84, P \ll 0.001)$. For small eccentricities, the extent of the surround mechanism, expressed as $2 \sigma$ of the inhibitory Gaussian envelope $\left(2 w_{s}\right)$, was $2.7^{\circ}$ and was well correlated with center mechanism width $(r=0.55, P \ll 0.001)$. Over all eccentricities, the ratio $w_{\mathrm{s}} / w_{\mathrm{c}}$ did not vary, with an overall mean of 2.5. This agrees with the results of Sceniak et al. (2001), who found that the surround mechanism in their DOG model was on average 2.2 times the width of the center mechanism, and with the related results of Levitt and Lund (2002).

Recall that the GSF grows approximately threefold at low contrast. We have now seen that the surround mechanism is about 2.5 times the width of the center mechanism. From these measurements, one might infer that the center and surround have similar extents. When we collected tuning curves for stimulus diameter at different stimulus contrasts, both stimulus contrast and stimulus diameter were variables. Rather than thinking of these data as revealing the recruitment of distal suppressive or excitatory areas depending on stimulus contrast, it is helpful to think of them instead as contrast responses for stimuli of different diameters (as in Fig. 8). When the stimulus is small, the center dominates, and a reduction in contrast causes a profound reduction in response. When the stimulus is large, more of the surround is being stimulated, and a reduction in contrast releases inhibition in the surround, yielding a smaller net reduction in response for larger stimuli. This diameter-dependent reduction in response is exactly what causes the shift in optimal stimulus diameter with contrast; this differential reduction can occur outside the central excitatory region. Therefore it is misleading to think of these data as showing how recruitment of excitatory or inhibitory regions depends on contrast. Rather they reveal the way that contrast changes the balance of center and surround signals.

Our grating measurements of receptive field extent relied on the assumption that receptive fields have circular spatial profiles. Although shapes of receptive fields do vary, the approximation of a circular receptive field is not unreasonable. DeAngelis et al. (1994) separately measured length- and widthtuning curves of receptive fields in cat area 17 and found substantial agreement between these two dimensions (see also Walker et al. 2000.). In the following paper (Cavanaugh et al. 2002), we address the issue of the specificity and spatial distribution of surround influence in more detail.

\section{Modeling center-surround interaction}

It is clear from the existence of surround suppression that neuronal responses depend on the interactions of inhibitory and excitatory receptive field components. Because responses are the product of excitatory and inhibitory influences, measurements of responses to high contrast stimuli alone are not sufficient to decipher the relative contributions of these influences, and therefore their extents remain uncertain. We thought that modeling the receptive field mechanisms as overlapping but independent Gaussians would permit us to translate a measurement of response as a function of stimulus diameter into an inference about the spatial extent of the actual receptive field mechanisms.

Our model employed an excitatory center mechanism with a broader, overlapping surround. One alternative model (Somers et al. 1998) accounts for surround suppression with a center and surround with identical spatial extents. However, this model implements surround suppression by changing the slope of a neuron's contrast response curve with surround stimulation. Our data indicate that the slope of this curve is the least affected feature of the contrast/response relationship, an observation corroborated by Sengpiel et al. (1998). Moreover, suppression from the similarly sized surround increases as contrast increases, but neither we nor Sceniak et al. (1999) observed any change in the degree of observed suppression with contrast. Because stimulating certain regions of the surround affects the inhibitory influence without affecting the center, we believe we are justified in assuming a broader inhibitory mechanism.

To construct a model with a center and a broader surround, we needed to know the manner in which the surround influences responses. Different types of suppression affect a neuron's contrast response differently. Heeger's normalization model (Heeger 1993) and Carandini and Heeger's extension of the basic normalization model (Carandini et al. 1997b) have been used to model the effects of changing a neuron's gain on its contrast response. The normalization model explains changes in a neuron's contrast response curve when an additional stimulus is placed within the receptive field. Adding a stimulus that is itself ineffective at driving the cell nevertheless suppresses responses to a preferred stimulus through divisive normalization contingent on stimulus contrast. We used a variant of the normalization model to determine that a divisive change in gain accounted for surround influences better than a subtractive form of suppression. For responses from within the receptive field of macaque V1 neurons, Carandini et al. (1997a) performed a similar analysis and concluded that changes in contrast gain were primarily responsible for these changes in the neuron's contrast response. Sengpiel et al. (1998) measured contrast response curves of a small population of cat cortical neurons in the presence of surround stimuli and found little difference between contrast gain and response gain fits to these curves. Because our results show that division more closely represented changes in contrast response and since previous research (DeAngelis et al. 1994; Sengpiel et al. 1998) suggested that the surround may work through divisive gain changes, we chose an RoG model.

Our results appear to be at variance with those of Anderson et al. (2001), who measured length summation in cat with intracellular recordings. Using a current-injection technique to separate excitatory and inhibitory influences, they found that the strength of center excitation decreased as grating patches were made large. However, their technique would only detect divisive inhibition if it took the form of a shunting input to the soma. If the gain control was achieved by other mechanisms (e.g., shunting inhibition in dendrites or circuits outside the neuron studied), the effect of our gain-setting surround would be exactly what they observed - the signal would not be evident as a frank inhibitory input at the soma but would nonetheless reduce the strength of excitatory inputs. In the context of our model, these results suggest that the mechanism of gain control by the surround is not captured by the simple singlecompartment model of Carandini et al. (1997b) but should be modeled with more realistic biophysics. 
Explaining changes in apparent receptive field size with gain changes

A number of groups have reported that the apparent size of the central region of cortical receptive fields can vary depending on the stimulation conditions and context. These findings have often been interpreted in terms of complex reorganizations of cortical circuits, but we believe that we can offer a simpler explanation. Pettet and Gilbert (1992) observed minimum response field expansion in primary visual cortex of cat after stimulating the CRF periphery with moving bars. Subsequently, Das and Gilbert (1995) observed minimum response field expansion in cat primary visual cortex when the surrounding area was stimulated with flashing dots. They postulated that the increase in MRF extent was due to an increase in connection strength between neurons, inferring that MRF expansion was associated with a change in the extent of effective connectivity within cortex. In addition, Kapadia et al. (1999) found excitation beyond the MRF when stimulating the surround with a high-contrast texture. We suggest that in these studies, instead of MRF expansion being the product of changes in cortical connectivity, the peripherally placed adapting stimuli or masks desensitized the surround of the receptive field and thereby increased the apparent size of the center (Fig. 7). This effect, however, is not seen when stimulating with high-contrast gratings, as the high-contrast grating drastically changes the gain of the center mechanism as well-more so than did the central bar stimuli used in Kapadia's experiment. Desensitization of the surround has the added benefit of explaining how some stimuli extending beyond the center mechanism could still result in a net increase of response, and how the GSF measured at low contrast could be larger than the width of the center mechanism. This serves to underscore the contrast between measurements of receptive field extent and the underlying mechanisms that produce them.

We have already noted our confirmation of the decrease in spatial summation with contrast that is a common feature of cortical responses (Das and Gilbert 1995; Kapadia et al. 1999; Sceniak et al. 1999). Previous reports have surmised that the large area of spatial integration at lower contrasts might also be due to an increase in the efficacy of lateral connections to other neurons when local contrast is low, resulting in a functional increase in the degree of lateral pooling by changes in connection strength. Our analysis suggests instead that a simple model in which only the gains of center and surround mechanisms vary can account well for changes in spatial summation with contrast. When we changed our model to permit the extent of the center mechanism to vary, we obtained no better an account of this phenomenon. We conclude that the variation of spatial summation with contrast and context is due to variations in the relative strength of the center and surround of the receptive field and that it is not necessary to postulate more elaborate changes in cortical connectivity to account for these effects.

\section{Cortical circuits that create the center and surround}

It is commonly considered that the classical receptive field reflects inputs that come primarily from neurons in the same column as the neuron being studied and that surround influences are carried by the network of horizontal intracortical connections (DeAngelis et al. 1994; Gilbert et al. 1996; Kapadia et al. 1999). But these ideas are based on MRF measure- ments of receptive field extent, measurements that we now know to be very conservative estimates of receptive field size. To determine the actual extent of the underlying receptive field mechanisms, we used the extent of the center mechanism in our model as a measure of the extent of the underlying neural substrate of the central excitatory portion of the receptive field. To compare our mechanism width with the underlying neural circuitry, we again defined the width of the central excitatory mechanism to be $2 \sigma$ of the Gaussian envelope of the center mechanism. For this comparison, we used model mechanism widths from the 55/79 cells that exhibited at least $10 \%$ suppression for high contrast stimuli.

We converted these widths into measures of cortical distance using cortical magnification factors fromTootell et al. (1988) and Van Essen et al. (1984). The distribution of mechanism diameters in units of cortical distance is shown in Fig. 13A.
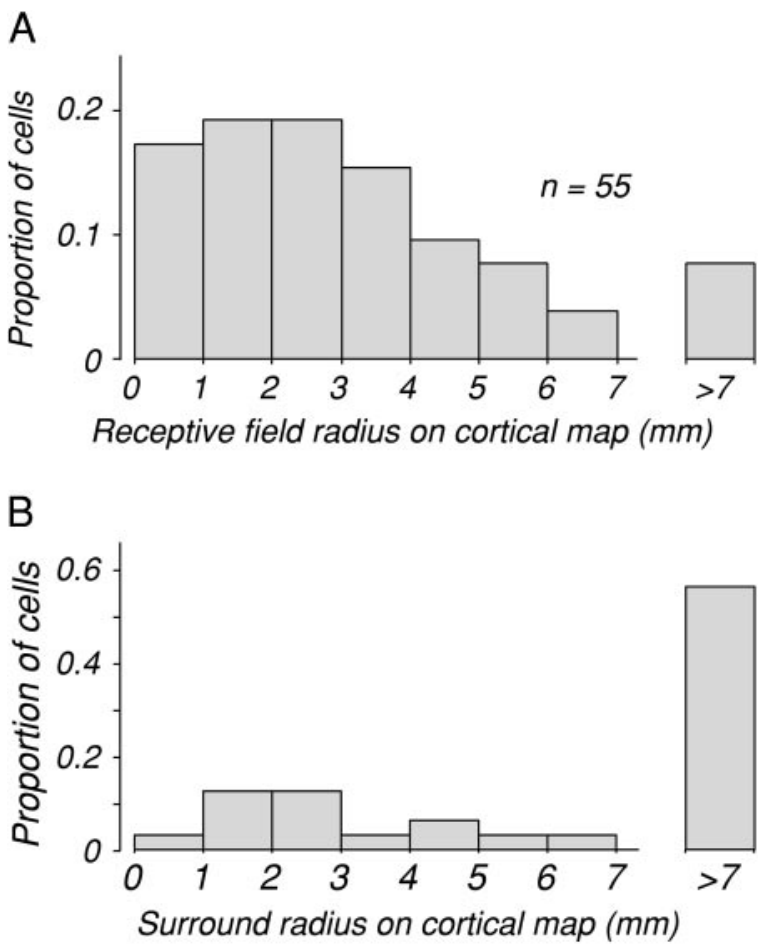

FIG. 13. Representation of center and surround extents on the cortex. We took RoG model center and surround mechanism widths from fits to diameter tuning curves at different contrasts (see Fig. 8). We used model parameters from cells showing $>10 \%$ suppression at high contrast (55/79 cells). We determined the width of each mechanism to be 2 times the SD of the Gaussian envelope (Fig. 6). We converted this mechanism width from degrees of visual angle into units of $\mathrm{mm}$ along cortex using the following approximate inverse cortical magnification factors

$$
c m f^{-1}= \begin{cases}0.15 E, & E<1^{\circ} \\ 0.0853+0.065 * E, & 1^{\circ} \leq E<4^{\circ} \\ 0.086 E, & E \geq 4^{\circ}\end{cases}
$$

where $\mathrm{cmf} f^{-1}$ is expressed in $\left(\mathrm{mm} /{ }^{\circ}\right)^{-1}$, and $E$ is receptive field eccentricity in degrees (Tootell et al. 1988; Van Essen et al. 1984). We have plotted the distributions of these cortical distances. A: center mechanism width expressed in $\mathrm{mm}$ of cortex. More than half of the receptive fields extend more than 2.5 $\mathrm{mm}$ along cortex - a distance similar to the typical length of horizontal intracortical connections (Yoshioka et al. 1996). This suggests that long-range horizontal connections link regions within the receptive field. B: surround mechanism width expressed in $\mathrm{mm}$ of cortex. The distribution of surround diameters is plotted on the same scale as center diameters. Even the longest estimates of horizontal connection length (Gilbert and Wiesel 1989) could not account for the degree of spatial summation deduced for surround influences in most neurons. 
Surprisingly, more than two-thirds of our neuronal receptive fields had diameters that exceeded $2 \mathrm{~mm}$ in cortical distance. Excitatory intracolumnar connections have a horizontal spread that is only a small fraction of this distance (Braitenburg and Schuz 1991), and the feature of cortical architecture that most closely matches it is the network of horizontal "long range" connections, which extend between 2 and $8 \mathrm{~mm}$ (Gilbert and Wiesel 1989; Yoshioka et al. 1996). We conclude that this network of connections, instead of carrying signals from topographically remote regions of $\mathrm{V} 1$, has the more mundane but still crucial function of providing the building blocks of cortical neurons' classical receptive fields. This also provides a ready explanation for the orientation specificity of horizontal connections (Gilbert and Wiesel 1989; Ts'o et al. 1986) because that specificity would ensure matched orientation selectivity throughout the classical receptive field.

But if horizontal connections create the classical receptive field, what is the origin of the signals that create the nonclassical surround? Figure $13 B$ uses the same mapping technique as Fig. $13 \mathrm{~A}$ to compute the distribution of cortical sizes of the surround mechanisms derived from our model. More than half of the surrounds have cortical extents greater than $7 \mathrm{~mm}$, at the very limit of the reported extent of horizontal intracortical connections. These data include cells for which there was no clear saturation of the suppressive influence for the largest high-contrast stimuli (23/55 cells) for which the determined surround extent is a lower bound. This would appear to rule out direct horizontal connections as a source of at least the more remote regions of the surround for most of our neurons. It seems likely that at least some of these signals originate in feedback signals from higher cortical areas. V1 receives strong feedback connections from several areas in which neurons have receptive fields large enough to provide these remote surround signals. Lund et al. (1999) have analyzed the divergence of feedforward and feedback connections, and their measurements confirm that feedback signals from V2, V3, and MT all have greater topographic spread than horizontal connections within V1 (see also Levitt and Lund 2002). Although feedback connections to V1 are most often described as excitatory (e.g., Hupé et al. 2001), inhibition is still possible if these connections terminate on inhibitory interneurons in V1. Not all of these extrastriate areas may be required for the generation of surround signals-for example, Hupé et al. (2001) have shown that surround suppression in V1 persists after inactivation of corresponding areas of V2. It nonetheless seems clear that surround signals in some V1 neurons arise from such remote parts of the visual field that it is implausible to suggest that they are carried by the system of horizontal intracortical connections alone. Some of these feedback connections are known to follow the same rules as intrinsic connections and link areas of like orientation selectivity (Gilbert and Wiesel 1989). Thus the specificity of feedback signals from higher cortical areas may determine the selectivity of the surround for stimulus features, an issue that is the subject of the following paper (Cavanaugh et al. 2002).

We are grateful to M. Hawken, P. Lennie, J. Levitt, and E. Simoncelli for advice and comments and to S. Fenstemaker for help with histology. M. A.
Smith and N. Majaj participated in some of the experiments. M. Sceniak shared some of unpublished data with us.

This work was supported by the Howard Hughes Medical Institute and by Project Grant EY02017 from the National Eye Institute to J. A. Movshon. J. R. Cavanaugh was supported by an National Science Foundation Predoctoral Fellowship and by a training grant from National Institutes of Health. W. Bair was partly supported by a grant from the Alfred P. Sloan Foundation.

\section{REFERENCES}

Albrecht DG ANd Hamilton DB. Striate cortex of monkey and cat: contrast response function. J Neurophysiol 48: 217-237, 1982.

Allman J, Miezin F, AND McGuinness E. Stimulus specific responses from beyond the classical receptive field: neurophysiological mechanisms for local-global comparisons in visual neurons. Аппи Rev Neurosci 8: 407-430, 1985.

Anderson JS, Lampl I, Gillespie DC, and Ferster D. Membrane potential and conductance changes underlying length tuning of cells in cat primary visual cortex. J Neurosci 21: 2104-2112, 2001.

Barlow HB, Blakemore C, and Pettigrew JD. The neural mechanism of binocular depth discrimination. J Physiol (Lond) 193: 327-342, 1967.

BLAKEMORE C AND TOBIN EA. Lateral inhibition between orientation detectors in the cat's visual cortex. Exp Brain Res 15: 439-440, 1972.

Braitenburg V and Schuz A. Anatomy of The Cortex. Statistics and Geometry. Berlin, Germany: Springer-Verlag, 1991:

Carandini M, Barlow HB, O'Keefe LP, Poirson AB, and Movshon JA. Adaptation to contingencies in macaque primary visual cortex. Philos Trans $R$ Soc Lond B Biol Sci 352: 1149-1154, 1997a.

CARANDINI M, HeEger DJ, AND Movshon JA. Linearity and normalization in simple cells of the macaque primary visual cortex. J Neurosci 17: 86218644, 1997b.

Cavanaugh JR, Bair W, AND Movshon JA. Selectivity and spatial distribution of signals from the receptive field surround in macaque V1 neurons. J Neurophysiol 88, 2547-2556, 2002.

Chandler JP. STEPIT. Indiana University Quantum Chemistry Program Exchange, 1965.

Chen CC, Kasamatsu T, Polat U, and Norcia AM. Contrast response characteristics of long-range lateral interactions in cat striate cortex. Neuroreport 12: 655-661, 2001.

DAS A AND GILBERT CD. Receptive field expansion in adult visual cortex is linked to dynamic changes in strength of cortical connections. $J$ Neurophysiol 74: 779-792, 1995.

DeAngelis GC, Freeman RD, AND OhZawa I. Length and width tuning of neurons in the cat's primary visual cortex. J Neurophysiol 71: 347-374, 1994

DREHER B. Hypercomplex cells in the cat's striate cortex. Invest Ophthalmol 11: 355-356, 1972.

Gilbert CD, Das A, Ito M, Kapadia M, and Westheimer G. Spatial integration and cortical dynamics. Proc Natl Acad Sci USA 93: 615-622, 1996.

GILBERT CD AND WIESEL TN. Columnar specificity of intrinsic horizontal and corticocortical connections in cat visual cortex. J Neurosci 9: 2432-2442, 1989.

HEEGER DJ. Modeling simple-cell direction selectivity with normalized, halfsquared, linear operators. J Neurophysiol 70: 1885-1898, 1993.

HoEl P, PORT S, AND STONE C. Introduction to Statistical Theory. Boston, MA: Houghton-Mifflin, 1971

Hubel DH AND Wiesel TN. Receptive fields and functional architecture of monkey striate cortex. J Physiol (Lond) 195: 215-243, 1968.

Hupé JM, JAMES AC, GiRARD P, AND Bullier J. Response modulations by static texture surround in area V1 of the macaque monkey do not depend on feedback connections from V2. J Neurophysiol 85: 146-163, 2001.

Kapadia MK, Ito M, Gilbert CD, and Westheimer G. Improvement in visual sensitivity by changes in local context: parallel studies in human observers and in V1 of alert monkeys. Neuron 15: 843-856, 1995.

Kapadia MK, Westheimer G, and Gilbert CD. Dynamics of spatial summation in primary visual cortex of alert monkeys. Proc Natl Acad Sci USA 96: 12073-12078, 1999.

LEVITT JB AND LUND JS. Contrast dependence of contextual effects in primate visual cortex. Nature 387: 73-76, 1997.

LEVITT JB AND LUND JS. The spatial extent over which neurons in macaque striate cortex pool visual signals. Vis Neurosci In press. 
LI CY AND LI W. Extensive integration field beyond the classical receptive field of cat's striate cortical neurons-classification and tuning properties. Vision Res 34: 2337-2355, 1994.

Lund JS, Angelucci A, Walton E, Bullier J, Hupé J, and Levitt JB. Topographic logic of connections within and between macaque monkey visual cortical areas V1, V2, V3, and V5. Inv Ophthalmol Vis Sci Supp 40: 645, 1999.

MAFFEI L AND FIORENTINI A. The unresponsive regions of visual cortical receptive fields. Vision Res 16: 1131-1139, 1976.

MERriLl EG AND Ainsworth A. Glass-coated platinum-plated tungsten microelectrodes. Med Biol Eng 10: 662-672, 1972.

Movshon JA, Thompson ID, AND Tolhurst DJ. Spatial summation in the receptive fields of simple cells in the cat's striate cortex. J Physiol (Lond) 283: 53-77, 1978a.

Movshon JA, Thompson ID, AND Tolhurst DJ. Receptive field organization of complex cells in the cat's striate cortex. J Physiol (Lond) 283: 79-99, $1978 b$.

NELSON JI AND FROST BJ. Intracortical facilitation among co-oriented, coaxially aligned simple cells in cat striate cortex. Exp Brain Res 61: 54-61, 1985.

Pettet MW and Gilbert CD. Dynamic changes in receptive-field size in cat primary visual cortex. Proc Natl Acad Sci USA 89: 8366-8370, 1992.

SCENIAK MP, HAWKEN MJ, AND SHAPLEY R. Visual spatial characterization of macaque v1 neurons. J Neurophysiol 85: 1873-1887, 2001.

SCEniaK MP, Ringach DL, Hawken MJ, AND ShaPley R. Contrast's effect on spatial summation by macaque V1 neurons. Nat Neurosci 2: 733-739, 1999.

SCHILlER PH, Finlay BL, AND Volman SF. Short-term response variability of monkey striate neurons. Brain Res 105: 347-349, 1976.

SClAR G, LENNIE P, AND DePRIEST DD. Contrast adaptation in striate cortex of macaque. Vision Res 29: 747-755, 1989.

Sengpiel F, Baddeley RJ, Freeman TC, Harrad R, and Blakemore C. Different mechanisms underlie three inhibitory phenomena in cat area 17. Vision Res 38: 2067-2080, 1998
Sillito AM, Grieve KL, Jones HE, CudeIRo J, AND DAVIS J. Visual cortical mechanisms detecting focal orientation discontinuities. Nature 378: 492 496, 1995.

Skottun BC, De Valois RL, Grosof DH, Movshon JA, Albrecht DG, And Bonds AB. Classifying simple and complex cells on the basis of response modulation. Vision Res 31: 1079-1086, 1991.

Somers DC, Todorov EV, Siapas AG, Toth LJ, Kim DS, AND Sur M. A local circuit approach to understanding integration of long-range inputs in primary visual cortex. Cereb Cortex 8: 204-217, 1998.

Tolhurst DJ, Movshon JA, AND ThOMPSON ID. The dependence of response amplitude and variance of cat visual cortical neurones on stimulus contrast. Exp Brain Res 41: 414-419, 1981.

Tolhurst DJ, Movshon JA, And DeAn AF. The statistical reliability of signals in single neurons in cat and monkey visual cortex. Vision Res 23: 775-785, 1983.

Tootell RB, Switkes E, Silverman MS, and Hamilton SL. Functional anatomy of macaque striate cortex. II. Retinotopic organization. J Neurosci 8: $1531-1568,1988$.

Ts'o DY, GILBERT CD, AND WIESEL TN. Relationships between horizontal interactions and functional architecture in cat striate cortex as revealed by cross-correlation analysis. J Neurosci 6: 1160-1170, 1986.

Van Essen DC, Newsome WT, And Maunsell JH. The visual field representation in striate cortex of the macaque monkey: asymmetries, anisotropies, and individual variability. Vision Res 24: 429-448, 1984.

VAUTIN RG AND BERKLEY MA. Responses of single cells in cat visual cortex to prolonged stimulus movement: neural correlates of visual aftereffects J Neurophysiol 40: 1051-1065, 1977.

Vogels R, SpILEers W, AND ORBAN GA. The response variability of striate cortical neurons in the behaving monkey. Exp Brain Res 77: 432-436, 1989.

Walker GA, Ohzawa I, AND FreEman RD. Suppression outside the classical cortical receptive field. Vis Neurosci 17: 369-379, 2000.

Yoshioka T, Blasdel GG, Levitt JB, And Lund JS. Relation between patterns of intrinsic lateral connectivity, ocular dominance, and cytochrome oxidase-reactive regions in macaque monkey striate cortex. Cereb Cortex 6: 297-310, 1996. 